\title{
Data-independent Low-complexity KLT Approximations for Image and Video Coding
}

\author{
A. P. Radünz * \\ T. L. T. da Silveira ${ }^{\dagger}$ \\ F. M. Bayer ${ }^{\ddagger}$ \\ R. J. Cintra $\S$
}

\begin{abstract}
The Karhunen-Loève transform (KLT) is often used for data decorrelation and dimensionality reduction. The KLT is able to optimally retain the signal energy in only few transform components, being mathematically suitable for image and video compression. However, in practice, because of its high computational cost and dependence on the input signal, its application in real-time scenarios is precluded. This work proposes low-computational cost approximations for the KLT. We focus on the blocklengths $N \in\{4,8,16,32\}$ because they are widely employed in image and video coding standards such as JPEG and high efficiency video coding (HEVC). Extensive computational experiments demonstrate the suitability of the proposed low-complexity transforms for image and video compression.
\end{abstract}

\section{Keywords}

Approximate transform, image compression, Karhunen-Loève transform, low-complexity transforms, signed KLT.

\section{Introduction}

The Karhunen-Loève transform (KLT) [1] is a commonly used tool for data decorrelation and dimensionality reduction [2,3]. It consists of a linear transformation that maps correlated variables into uncorrelated variables, sometimes referred to as principal components [4]. Usually, only the first coefficients of the transformed data are sufficient to represent the signal. The KLT capability for energy compaction is paramount for data compression, since most information can be preserved even reducing the dimensionality of the data [5]. In fact, considering first-order Markov processes, the KLT is an optimal linear transform capable of minimizing the mean square error in data compression and concentrating energy in few coefficients of the output signal [1]. Although it is a wellestablished optimal transform in terms of energy compaction and decorrelation [6], the KLT is not widely applied because its computation depends on the covariance matrix of the input data. Indeed, such data-dependent requirements can hinder the development of fast algorithms for an efficient implementation of the transform.

However, if the input data is a first-order Markov process with known correlation coefficient $\rho$, then it was shown in [7] that we can derive an analytical solution for the elements of the KLT matrix. Nevertheless, even with the transform matrix known, computational complexity of its implementation can be infeasible for practical data compression scenarios. In this context, several fast approximations for the KLT have been proposed [8-17] aiming at reducing the computational costs. Although such methods generate fast approximations for the KLT, their scope is relatively limited because the data-dependence is still present; in some cases either depending on the covariance matrix of the input data [9-13 15, 17] or on the correlation coefficient in case of first-order Markovian signals [8].

When considering first-order Markovian random signals, [18] and [19,20] have shown that the discrete cosine transform (DCTII) and the discrete sine transform (DST-I) are asymptotic approximations for the KLT, with the correlation coefficient of the input signal tending to unity and to zero, respectively [18]. Both the DCT and the DST are independent of the input signal, allowing

*Programa de Pós-Graduação em Estatística, Universidade Federal de Pernambuco, Recife, Brazil. E-mail: apr1@de.ufpe.br

${ }^{\dagger}$ Instituto de Informática, Universidade Federal do Rio Grande do Sul, Porto Alegre, Brazil. E-mail: thiago@inf.ufsm.br

‡Departamento de Estatística and LACESM, Universidade Federal de Santa Maria, Santa Maria, Brazil. E-mail:|bayer@ufsm.br

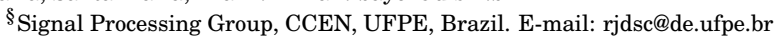

the development of computationally efficient fast algorithms. The DCT is widely adopted in image and video compression standards such as JPEG [21] and high efficiency video coding (HEVC) [22], just to name a few. However, the use of this transform can still be prohibitive in contexts under severe restrictions on processing power or energy autonomy [23-26]. In fact, DCT realizations that require multiplications implemented in floating-point arithmeticbased hardware |27| demand significant circuitry complexity and energy consumption [1]. In this sense, several multiplication-free approximations for the DCT have been proposed [23, 25, 28, 38], including the signed DCT (SDCT) [39]. The SDCT is derived by applying the signum function to the elements of the DCT matrix, thus resulting in a matrix of trivial multiplicands $\{-1,+1\}$. Therefore, the transform computation requires only additions. Such reduction in the arithmetic cost implies in a lower computational cost, favoring applications in real-time and in low-consumption devices [1].

The present work employs the signum function as a means to obtain computationally efficient alternatives to the KLT for firstorder Markov processes. We follow an entirely different approach when compared with the fast KLT approximations already known in the literature. Here, we focus on the proposition of deterministically defined multiplierless low-complexity approximations for the KLT that does not depend on the input signal and is capable of coping with a wide range of correlation coefficients. Our analyses are devoted to the blocklengths $N \in\{4,8,16,32\}$ because of their relevance in image and video standards as JPEG [21] and HEVC [22]. In order to find the best-performing low-cost approximations, we propose a constrained optimization approach according to suitable figures of merit for the KLT analysis. The considered approximation method is specifically tailored to furnish low-complexity transformations appropriate for dedicated highly-efficient circuitry design. The resulting KLT approximations are sought to be numerically evaluated according to coding performance [40 41], and similarity/proximity metrics [1, 28] with respect to the exact KLT. The obtained transforms are then embedded into (i) a JPEG-like image compression scheme, and (ii) an HEVC reference software for video coding assessment.

To the best of our knowledge, literature lacks KLT approximations that combine the following properties:

1. deterministic definition;

2. suitability for fast algorithm design;

3. data-independence; and 
4. capability of processing data at a wide range correlation.

We aim, therefore, at a proposition of a new class of KLT approximations that addresses these gaps. The main goal of our paper is to propose low-complexity approximate transforms for the KLT considering different values of the correlation coefficients $\rho$, so low and mid-correlated signals could be properly treated as well. Since, to the best of our knowledge, the literature lacks efficient KLT-based methods considering lowly correlated data, there is no competing method for a fair comparison.

This paper is structured as follows. In Section 2, we revise the mathematical formulation of the KLT for first-order Markovian signals and define the general framework for signed KLT (SKLT). Section 3 describes the computational approach for obtaining new transforms and presents these transforms for different lengths attaining optimality according to the proposed figures of merit based on classical metrics. Section 3 also presents fast algorithms for the proposed transforms. In Section 4 we assess the proposed 4-, 8-, $16-$, and 32-point SKLT in image and video coding. Section 5 concludes the paper.

\section{Signed KLT}

\subsection{Karhunen-Loève Transform for the First-order Markov Process}

The KLT is a linear transformation represented by an orthogonal matrix $\mathbf{K}_{N}^{(\rho)}$ which decorrelates an input signal $\mathbf{x}=$ $\left[\begin{array}{llll}x_{0} & x_{1} & \ldots & x_{N-1}\end{array}\right]^{\top}$ resulting in uncorrelated signal $\mathbf{y}=$ $\left[\begin{array}{llll}y_{0} & y_{1} & \ldots & y_{N-1}\end{array}\right]^{\top}$. The $(i, j)$ th elements of the transform matrix $\mathbf{K}_{N}^{(\rho)}$, for an arbitrary value of $\rho \in[0,1]$, are given by $[1]$

$$
\begin{array}{r}
k_{i j}=\sqrt{\frac{2}{N+\lambda_{j}}} \sin \left[\omega_{j}\left(i-\frac{N-1}{2}\right)+\frac{(j+1) \pi}{2}\right], \\
i, j=0,1, \ldots, N-1,
\end{array}
$$

where the eigenvalues of the transformed signal $\mathbf{y}$ covariance matrix are obtained by

$$
\lambda_{j}=\frac{1-\rho^{2}}{1+\rho^{2}-2 \rho \cos \omega_{j}}, \quad j=0,1, \ldots, N-1,
$$

and $\omega_{1}, \omega_{2}, \ldots, \omega_{N}$ are the $N$ solutions of the non-linear equation

$$
\tan N \omega=\frac{-\left(1-\rho^{2}\right) \sin \omega}{\left(1+\rho^{2}\right) \cos \omega-2 \rho}
$$

It is a well-known fact that adjacent pixels from natural images are highly correlated [42], being $\rho=0.95$ a widely adopted assumption [1]. When the correlation of the input signal tends the unity, $\rho \rightarrow 1$, the KLT converges to the DCT [18].

For instance, if $N=8$ and $\rho=0.95$, then the KLT matrix is given by

$$
\mathbf{K}_{8}^{(0.95)}=\left[\begin{array}{rrrrrrrr}
0.338 & 0.351 & 0.360 & 0.364 & 0.364 & 0.360 & 0.351 & 0.338 \\
0.481 & 0.420 & 0.286 & 0.101 & -0.101 & -0.286 & -0.420 & -0.481 \\
0.467 & 0.207 & -0.179 & -0.456 & -0.456 & -0.179 & 0.207 & 0.467 \\
0.423 & -0.085 & -0.487 & -0.278 & 0.278 & 0.487 & 0.085 & -0.423 \\
0.360 & -0.347 & -0.356 & 0.351 & 0.351 & -0.356 & -0.347 & 0.360 \\
0.283 & -0.488 & 0.094 & 0.415 & -0.415 & -0.094 & 0.488 & -0.283 \\
0.195 & -0.462 & 0.460 & -0.190 & -0.190 & 0.460 & -0.462 & 0.195 \\
0.100 & -0.279 & 0.416 & -0.490 & 0.490 & -0.416 & 0.279 & -0.100
\end{array}\right]
$$

\subsection{KLT Approximations}

Our approach is based on the technique used in [39] for proposing the classical signed DCT (SDCT). The proposed transform, as well as the SDCT, is motivated by the reduction of the total number of arithmetic operations required for the computation of the transform at the cost of some accuracy loss [39]. The technique considers the signum function to generate a matrix approximation for the KLT. Thus, we propose the following approximate transformation matrix:

$$
\widehat{\mathbf{T}}_{N}^{(\rho)} \triangleq \frac{1}{\sqrt{N}} \operatorname{sign}\left(\mathbf{K}_{N}^{(\rho)}\right),
$$

where

$$
\operatorname{sign}(x)= \begin{cases}1, & \text { if } x>0 \\ 0, & \text { if } x=0 \\ -1, & \text { if } x<0\end{cases}
$$

and $\mathbf{K}_{N}^{(\rho)}$ is the KLT matrix of order $N$ with a predefined correlation coefficient $\rho$, which entries are given by (1). When applied to a matrix, the signum function operates element-wise.

In other words, we map a given KLT matrix to a low-complexity matrix close to it. Note that, if $\rho=0$, then the Equation (5) degenerates into the null matrix. Also if $\rho=1$, then $\mathbf{K}_{N}^{(1)}$ is the DCT and the resulting approximation is the signed DCT [39]. Therefore, in practice, our analysis is constrained to $0<\rho<1$. Note that because of the non-linearity and discrete nature of the signum function, different KLT matrices might be mapped to the same approximate matrix. For instance, considering $\rho_{1}=0.7$ and $\rho_{2}=0.9$, we obtain that

$$
\widehat{\mathbf{T}}_{8}^{\left(\rho_{1}\right)}=\widehat{\mathbf{T}}_{8}^{\left(\rho_{2}\right)}=\frac{1}{\sqrt{8}}\left[\begin{array}{rrrrrrrr}
1 & 1 & 1 & 1 & 1 & 1 & 1 & 1 \\
1 & 1 & 1 & 1 & -1 & -1 & -1 & -1 \\
1 & 1 & -1 & -1 & -1 & -1 & 1 & 1 \\
1 & -1 & -1 & -1 & 1 & 1 & 1 & -1 \\
1 & -1 & -1 & 1 & 1 & -1 & -1 & 1 \\
1 & -1 & 1 & 1 & -1 & 1 & 1 & -1 \\
1 & -1 & 1 & -1 & -1 & 1 & -1 & 1 \\
1 & -1 & 1 & -1 & 1 & -1 & 1 & -1
\end{array}\right] .
$$

Exhaustively computing all KLT matrices in the range $\rho \in$ $\left[10^{-3}, 1-10^{-3}\right]$ in steps of $10^{-3}$ returns 999 matrices. However, the number of approximations is much lower: 1, 2, 9, and 37 different approximations for $N=4,8,16$, and 32 , respectively. In view of the above, a methodology for selecting best-performing approximations is necessary, which is the topic of the next section.

Although the KLT is an orthogonal matrix, the proposed transforms are not constrained to be, therefore, given the proposed transform $\widehat{\mathbf{T}}_{N}^{(\rho)}$, the transformed signal is given by

$$
\mathbf{y}=\widehat{\mathbf{T}}_{N}^{(\rho)} \cdot \mathbf{x},
$$

and the inverse transformation can be written as

$$
\mathbf{y}=\left(\widehat{\mathbf{T}}_{N}^{(\rho)}\right)^{-1} \cdot \mathbf{x}
$$

\section{Optimal SKLT}

In this section, we describe an optimization problem, aiming at the identification of best-performing SKLT matrices, according to the figures of merit detailed next.

\subsection{Figures of Merit for Approximate Transforms}

Approximate transform methods [23, 25, 28, 39] are usually assessed in terms of (i) coding metrics such as the coding gain [40] and transform efficiency [41], which measure the power of decorrelation and energy compression; and (ii) proximity metrics with respect to the exact transform, such as the mean-square error [1] and 
total error energy [28], which measure similarities or dissimilarities between approximate and exact transforms. In the following, let $\widehat{\mathbf{T}}_{N}$ be a candidate matrix to be assessed.

\subsubsection{Unified Coding Gain}

The unified coding gain of a transform $\widehat{\mathbf{T}}_{N}$ is given by 43

$$
\operatorname{Cg}\left(\widehat{\mathbf{T}}_{N}\right)=10 \cdot \log _{10}\left\{\prod_{k=1}^{N} \frac{1}{\sqrt[N]{A_{k} \cdot B_{k}}}\right\}
$$

where $A_{k}=\operatorname{su}\left\{\left(\mathbf{h}_{k}^{\top} \cdot \mathbf{h}_{k}\right) \odot \mathbf{R}_{\mathbf{x}}\right\}, \mathbf{h}_{k}$ is the $k$ th row vector from $\widehat{\mathbf{T}}_{N}$, the function $\mathrm{su}(\cdot)$ returns the sum of the elements of its matrix argument, $\odot$ is the Hadamard matrix product operator [44], $\mathbf{R}_{\mathbf{x}}$ is the autocorrelation matrix of the considered first-order Markovian signal, $B_{k}=\left\|\mathbf{g}_{k}\right\|^{2}$ and $\mathbf{g}_{k}$ is the $k$ th row vector from $\widehat{\mathbf{T}}_{N}^{-1}$, and $\|\cdot\|$ is the Frobenius norm [44].

\subsubsection{Transform Efficiency}

Another coding related figure of merit is the transform efficiency, given by [41]

$$
\eta\left(\widehat{\mathbf{T}}_{N}\right)=100 \frac{\sum_{i=1}^{N}\left|r_{i, i}\right|}{\sum_{i=1}^{N} \sum_{j=1}^{N}\left|r_{i, j}\right|},
$$

where $r_{i, j}$ is the $(i, j)$ th element from $\widehat{\mathbf{T}}_{N} \cdot \mathbf{R}_{\mathbf{x}} \cdot \widehat{\mathbf{T}}_{N}^{\top}$.

\subsubsection{Mean-Square Error}

The mean-square error (MSE) relative to the KLT is given by [1]:

$$
\operatorname{MSE}\left(\widehat{\mathbf{T}}_{N}\right)=\frac{1}{N} \cdot \operatorname{tr}\left\{\left(\mathbf{K}_{N}^{(\rho)}-\widehat{\mathbf{T}}_{N}\right) \cdot \mathbf{R}_{\mathbf{x}} \cdot\left(\mathbf{K}_{N}^{(\rho)}-\widehat{\mathbf{T}}_{N}\right)^{\top}\right\}
$$

where $\operatorname{tr}(\cdot)$ is the trace function [45].

\subsubsection{Total Error Energy}

The total error energy of an approximation relative to the KLT is computed by [28]:

$$
\epsilon\left(\widehat{\mathbf{T}}_{N}\right)=\pi \cdot\left\|\mathbf{K}_{N}^{(\rho)}-\widehat{\mathbf{T}}_{N}\right\|^{2}
$$

\subsubsection{Proposed Figures of Merit}

Because the above discussed figures of merit are defined for a fixed value of $\rho$, we propose the following total metrics which take into account the performance for all values of $0<\rho<1$ :

$$
\begin{aligned}
\operatorname{Cg}_{T}\left(\widehat{\mathbf{T}}_{N}\right) & =\int_{0}^{1}\left|\operatorname{Cg}\left(\mathbf{K}_{N}^{(\rho)}\right)-\operatorname{Cg}\left(\widehat{\mathbf{T}}_{N}\right)\right| \mathrm{d} \rho \\
\eta_{T}\left(\widehat{\mathbf{T}}_{N}\right) & =\int_{0}^{1}\left|\eta\left(\mathbf{K}_{N}^{(\rho)}\right)-\eta\left(\widehat{\mathbf{T}}_{N}\right)\right| \mathrm{d} \rho \\
\operatorname{MSE}_{T}\left(\widehat{\mathbf{T}}_{N}\right) & =\int_{0}^{1} \operatorname{MSE}\left(\widehat{\mathbf{T}}_{N}\right) \mathrm{d} \rho \\
\epsilon_{T}\left(\widehat{\mathbf{T}}_{N}\right) & =\int_{0}^{1} \epsilon\left(\widehat{\mathbf{T}}_{N}\right) \mathrm{d} \rho .
\end{aligned}
$$

\subsection{Optimization Problem}

In order to identify the overall best-performing approximations, we propose the following optimization problem:

$$
\widehat{\mathbf{T}}_{N}^{*}=\underset{0<\rho<1}{\arg \min \operatorname{error}}\left(\widehat{\mathbf{T}}_{N}^{(\rho)}\right),
$$

where error( $(\cdot)$ is one of the proposed measures, $\operatorname{Cg}_{T}(\cdot), \eta_{T}(\cdot)$,

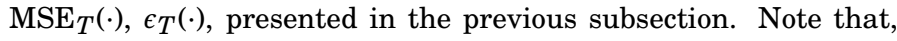
for a fixed transform length $N$, up to four optimal SKLT can be obtained, each one optimizing a total metric. Hereafter we denote the optimal transforms of length $N \in\{4,8,16,32\}$ as $\widehat{\mathbf{T}}_{N, i}$, which are indexed by the subscript $i=1,2, \ldots, J$, where $J$ is the number of approximate transforms for $N$.

Table 1 summarizes the results for the optimal SKLT with different transform lengths $N$ and for the intervals of $\rho$ that each transform is defined. DCT, DST, and the SDCT results are included only for comparison purposes. It is important to emphasize again that this comparison is not completely fair since the proposed transforms cover different intervals of $\rho$ while the DCT and DST are defined for $\rho$ tending merely to one and zero, respectively. All metrics are computed with respect to the exact KLT. The values in bold are the best measurements for each transform length $N$. The transforms $\widehat{\mathbf{T}}_{4,1}, \widehat{\mathbf{T}}_{8,2}$, and $\widehat{\mathbf{T}}_{16,3}$ are already known in the literature, and coincide with the SDCT. The remaining transforms are, to the best of our knowledge, new ones.

\subsection{Fast Algorithms}

The direct implementation of the proposed transforms requires $N(N-1)$ additions and no multiplications. Besides searching for multiplication-free transforms, the development of fast algorithms capable of reducing the arithmetic cost of computing the transforms is important. Using sparse matrix factorization [46], such as butterfly-based structures, we can derive the factorization for the optimal proposed transforms. In order to assess the complexity of the proposed fast algorithms we considered the number of arithmetic operations needed for its implementation. The arithmetic complexity does not depend on the available architecture or technology, an issue that may occur when considering measures such as computation time [46-48]. The derived matrix factorization for $N=4$ and 8 are presented in the following, and for $N=16$ and 32, the respective matrix factorization are detailed in Appendix $\mathrm{A}$

\subsubsection{Matrix Factorization for $N=4$}

For $N=4$, we can factorize $\widehat{\mathbf{T}}_{4,1}|39|$ as follows:

$$
\widehat{\mathbf{T}}_{4,1}=\frac{1}{2} \cdot \mathbf{P}_{4} \cdot \mathbf{A}_{4,2} \cdot \mathbf{A}_{4,1},
$$

where

$$
\mathbf{P}_{4}=\left[\begin{array}{llll}
1 & & & \\
& & 1 & \\
& 1 & & \\
& & & 1
\end{array}\right], \quad \mathbf{A}_{4,2}=\left[\begin{array}{cccc}
1 & 1 & & \\
1 & - & & \\
& & 1 & 1 \\
& & - & 1
\end{array}\right],
$$

and

$$
\mathbf{A}_{4,1}=\left[\begin{array}{cc}
\mathbf{I}_{2} & \overline{\mathbf{I}}_{2} \\
\overline{\mathbf{I}}_{2} & -\mathbf{I}_{2}
\end{array}\right]
$$

where $\mathbf{I}_{2}$ and $\overline{\mathbf{I}}_{2}$ are, respectively, the identity and counter-identity matrices of order 2 . The minus sign - represents -1 and blank spaces are zeroes. 
Table 1: Comparison of proposed transforms with KLT

\begin{tabular}{|c|c|c|c|c|c|}
\hline Transform & $\rho$ & $\mathrm{Cg}_{T}\left(\widehat{\mathbf{T}}_{N}\right)$ & $\eta_{T}\left(\widehat{\mathbf{T}}_{N}\right)$ & $\operatorname{MSE}_{T}\left(\widehat{\mathbf{T}}_{N}\right)$ & $\epsilon_{T}\left(\widehat{\mathbf{T}}_{N}\right)$ \\
\hline \multicolumn{6}{|c|}{$N=4$} \\
\hline$\widehat{\mathbf{T}}_{4,1} \mid 39$ & $(0,1)$ & 0.162 & 10.862 & 0.039 & 0.764 \\
\hline $\mathrm{DCT}$ & $\rho \rightarrow 1$ & 0.025 & 7.071 & 0.014 & 0.167 \\
\hline DST & $\rho \rightarrow 0$ & 0.317 & 11.859 & 0.016 & 0.167 \\
\hline \multicolumn{6}{|c|}{$N=8$} \\
\hline$\widehat{\mathbf{T}}_{8,1}$ & $(0,0.619]$ & 2.726 & 32.907 & 0.110 & 3.670 \\
\hline$\widehat{\mathbf{T}}_{8,2} \mid 39$ & $(0.619,1)$ & 2.170 & 37.192 & 0.129 & 3.950 \\
\hline $\mathrm{DCT}$ & $\rho \rightarrow 1$ & 0.031 & 12.173 & 0.042 & 0.888 \\
\hline DST & $\rho \rightarrow 0$ & 0.362 & 18.940 & 0.032 & 0.778 \\
\hline \multicolumn{6}{|c|}{$N=16$} \\
\hline$\widehat{\mathbf{T}}_{16,1}$ & $(0.540,0.550]$ & 2.825 & 56.924 & 0.157 & 8.499 \\
\hline$\widehat{\mathbf{T}}_{16,2}$ & $(0.550,0.700]$ & 2.748 & 54.668 & 0.153 & 8.538 \\
\hline$\widehat{\mathbf{T}}_{16,3}$ & {$[0.9,1)$} & 2.227 & 48.588 & 0.169 & 9.532 \\
\hline DCT & $\rho \rightarrow 1$ & 0.024 & 15.709 & 0.078 & 2.945 \\
\hline DST & $\rho \rightarrow 0$ & 0.307 & 22.772 & 0.044 & 2.283 \\
\hline \multicolumn{6}{|c|}{$N=32$} \\
\hline$\widehat{\mathbf{T}}_{32,1}$ & $(0.139,0.162]$ & 2.352 & 66.355 & 0.195 & 21.143 \\
\hline$\widehat{\mathbf{T}}_{32,2}$ & $(0.487,0.490]$ & 2.442 & 65.047 & 0.184 & 19.806 \\
\hline$\widehat{\mathbf{T}}_{32,3}$ & $(0.490,0.528]$ & 2.477 & 65.475 & 0.185 & 19.779 \\
\hline$\widehat{\mathbf{T}}_{32,4}$ & $(0.956,0.977]$ & 2.450 & 61.930 & 0.244 & 24.288 \\
\hline SDCT & $\rho \rightarrow 1$ & 2.491 & 62.550 & 0.256 & 24.856 \\
\hline DCT & $\rho \rightarrow 1$ & 0.015 & 18.005 & 0.116 & 7.891 \\
\hline DST & $\rho \rightarrow 0$ & 0.227 & 24.673 & 0.052 & 5.531 \\
\hline
\end{tabular}

\subsubsection{Matrix Factorization for $N=8$}

For $N=8$, we have:

$$
\begin{aligned}
& \widehat{\mathbf{T}}_{8,1}=\frac{1}{\sqrt{8}} \cdot \mathbf{P}_{8} \cdot \mathbf{A}_{8,3}^{\prime} \cdot \mathbf{A}_{8,2} \cdot \mathbf{A}_{8,1}, \\
& \widehat{\mathbf{T}}_{8,2}=\frac{1}{\sqrt{8}} \cdot \mathbf{P}_{8} \cdot \mathbf{A}_{8,3}^{\prime \prime} \cdot \mathbf{A}_{8,2} \cdot \mathbf{A}_{8,1}[39,
\end{aligned}
$$

where

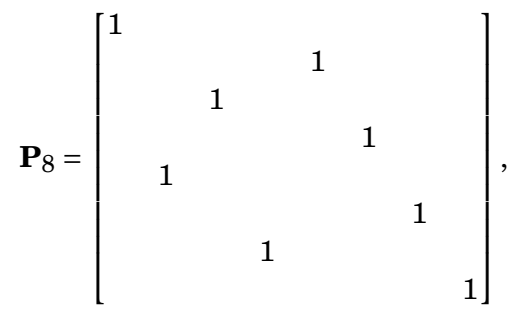

$$
\begin{aligned}
& \mathbf{A}_{8,3}^{\prime}=\left[\begin{array}{cccccccc}
1 & 1 & & & & & & \\
1 & - & & & & & & \\
& & 1 & 1 & & & & \\
& & - & 1 & & & & \\
& & & & 1 & 1 & & \\
& & & & & & - & - \\
& & & & 1 & & 1 & \\
& & & & & & 1 & -
\end{array}\right] \\
& \mathbf{A}_{8,2}=\left[\begin{array}{ll}
\mathbf{A}_{4,1} & \\
& \mathbf{A}_{4,1}
\end{array}\right], \quad \mathbf{A}_{8,1}=\left[\begin{array}{cc}
\mathbf{I}_{4} & \overline{\mathbf{I}}_{4} \\
\overline{\mathbf{I}}_{4} & -\mathbf{I}_{4}
\end{array}\right],
\end{aligned}
$$

and

$$
\mathbf{A}_{8,3}^{\prime \prime}=\left[\begin{array}{ccccccccc}
1 & 1 & & & & & & \\
1 & - & & & & & & \\
& & 1 & 1 & & & & \\
& & 1 & - & & & & \\
& & & & 1 & 1 & & \\
& & & & & - & & - \\
& & & & 1 & & 1 & \\
& & & & & & 1 & -
\end{array}\right] .
$$

Table 2 presents the arithmetic cost of the proposed fast algorithms for the approximate transforms compared with the arithmetic cost of the direct implementation of the exact $N$-point KLT. Since the proposed transforms are, to the best of our knowledge, the first class of approximations for the KLT following this approach, there is nothing to compare with. However, to show that the performance of the proposed transforms is competitive, we introduced in Table 3 the arithmetic cost of some transforms in general that are already known in the literature. Thus, Table 3 presents the arithmetical cost of: the fast algorithms proposed by [49] and [50] for the 8- and 16-point DCT, respectevely; and approximations for the DCT already known in the literature proposed in |25 28, 31 33 39 51, 56]. We can note that the introduced fast algorithms are multiplierless and offer substantial reductions in the additive complexity when compared with the exact KLT and lower or similar arithmetic cost compared with the DCT approximations.

For a better visualization of the results presented in Tables 1 and 2 we have combined graphically the number of additions and the proposed figures of merit of each proposed transform, for $N=4$, 8, 16, and 32, as presented in Figures 1, 2, 3, respectively. Note that both DCT and DST also require multiplications when imple- 
Table 2: Computational cost comparison for 4-, 8-, 16-, and 32-point transforms

\begin{tabular}{|c|c|c|c|c|}
\hline Transform & Additions & Multiplications & Bit-Shifting & Addition reduction (\%) \\
\hline$\widehat{\mathbf{T}}_{4,1} \mid 39$ & 4 & 0 & 0 & $66 \%$ \\
\hline $\mathbf{K}_{4}$ & 12 & 16 & 0 & - \\
\hline$\widehat{\mathbf{T}}_{8,1}$ & 24 & 0 & 0 & $57 \%$ \\
\hline$\widehat{\mathbf{T}}_{8,2}|39|$ & 24 & 0 & 0 & $57 \%$ \\
\hline $\mathbf{K}_{8}$ & 56 & 64 & 0 & - \\
\hline$\widehat{\mathbf{T}}_{16,1}$ & 80 & 0 & 0 & $67 \%$ \\
\hline$\widehat{\mathbf{T}}_{16,2}$ & 75 & 0 & 0 & $69 \%$ \\
\hline$\widehat{\mathbf{T}}_{16,3}|39|$ & 72 & 0 & 0 & $70 \%$ \\
\hline $\mathbf{K}_{16}$ & 240 & 256 & 0 & - \\
\hline$\widehat{\mathbf{T}}_{32,1}$ & 288 & 0 & 0 & $71 \%$ \\
\hline$\widehat{\mathbf{T}}_{32,2}$ & 288 & 0 & 0 & $71 \%$ \\
\hline$\widehat{\mathbf{T}}_{32,3}$ & 288 & 0 & 0 & $71 \%$ \\
\hline$\widehat{\mathbf{T}}_{32,4}$ & 232 & 0 & 0 & $77 \%$ \\
\hline $\mathbf{K}_{32}$ & 992 & 1024 & 0 & - \\
\hline
\end{tabular}

Table 3: Computational cost comparison for 8-, 16-, and 32-point DCT approximation transforms

\begin{tabular}{|c|c|c|c|}
\hline Transform & Additions & Multiplications & Bit-Shifting \\
\hline $\mathrm{DCT}_{8}[49$ & 29 & 11 & 0 \\
\hline $\mathrm{DST}_{8} \mid \overline{57}$ & 29 & 12 & 0 \\
\hline $\left.\mathbf{T}_{8, \mathrm{LO}} \mid \overline{31}\right]$ & 24 & 0 & 2 \\
\hline $\mathbf{T}_{8, \mathrm{RDCT}}|28|$ & 22 & 0 & 0 \\
\hline $\mathbf{T}_{8, \mathrm{MRDCT}}|\overline{52}|$ & 14 & 0 & 0 \\
\hline $\mathbf{T}_{8, \mathrm{BAS}-2008 \mathrm{a}}$ & 18 & 0 & 2 \\
\hline $\mathbf{T}_{8, \mathrm{BAS}-2008 \mathrm{~b}} \mid \overline{25}$ & 21 & 0 & 2 \\
\hline $\mathbf{T}_{8, \mathrm{BAS}-2009}$ & 18 & 0 & 0 \\
\hline 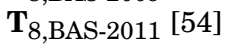 & 16 & 0 & 0 \\
\hline $\mathbf{T}_{8, \mathrm{BAS}-2012}|\overline{29}|$ & 24 & 0 & 0 \\
\hline $\mathbf{T}_{8,1}^{\prime}[55$ & 18 & 0 & 0 \\
\hline $\mathbf{T}_{8,4} \mid \overline{55}$ & 24 & 0 & 0 \\
\hline $\mathbf{T}_{8,5} \mid \overline{55}$ & 24 & 0 & 4 \\
\hline $\mathbf{T}_{8,6}|\overline{55}|$ & 24 & 0 & 6 \\
\hline $\mathrm{DCT}_{16}$ & 74 & 44 & 0 \\
\hline $\mathrm{DST}_{16}|\overline{57}|$ & 81 & 32 & 0 \\
\hline $\mathbf{T}_{16, \text { BAS-2012 }}$ & 64 & 0 & 0 \\
\hline $\mathbf{T}_{16, \mathrm{BCEM}} \mid 51$ & 72 & 0 & 0 \\
\hline $\mathbf{T}_{16, \text { SBCKMK }} \mid 56$ & 60 & 0 & 0 \\
\hline $\mathbf{T}_{16, \text { SOBCM }}$ & 44 & 0 & 0 \\
\hline $\left.\mathbf{T}_{16, \mathrm{JAM}} \mid 30\right]$ & 60 & 0 & 0 \\
\hline $\mathrm{DCT}_{32}$ & 194 & 116 & 0 \\
\hline $\mathrm{DST}_{32}|\overline{57}|$ & 209 & 80 & 0 \\
\hline $\mathbf{T}_{32, \mathrm{BAS}-2012}[29]$ & 160 & 0 & 0 \\
\hline $\left.\mathbf{T}_{32, \mathrm{JAM}} \mid 30\right]$ & 152 & 0 & 0 \\
\hline
\end{tabular}

mented, differently of the proposed transforms that are multiplierless.

\section{Image and Video Coding}

We submitted the proposed transforms to two different contexts that are classical in the approximation community area: (i) still image compression according to a JPEG-like algorithm [23 25 28], and (ii) video encoding as defined in the HEVC reference software [58]. In this section, we compared the proposed transforms with the exact KLT for $\rho=0.1,0.6$ and $0.9\left(\mathbf{K}^{(0.1)}, \mathbf{K}^{(0.6)}\right.$ and $\left.\mathbf{K}^{(0.9)}\right)$, and with the exact DCT. The transforms $\mathbf{K}^{(0.1)}, \mathbf{K}^{(0.6)}$, and $\mathbf{K}^{(0.9)}$ were selected because they are suitable for decorrelation of lowly, moderately, and highly correlated data, respectively. Currently literature is restricted to the high correlation scenario, which is mainly addressed by the DCT and its related approximations.

\subsection{Image Compression}

In this section, we evaluated the performance of the proposed transforms in image compression, similarly to [23 25, 28]. If $\mathbf{A}$ is a two-dimensional (2D) image, then the direct and inverse transformations induced by the SKLT are computed, respectively, by

$$
\begin{aligned}
& \mathbf{B}=\widehat{\mathbf{T}}_{N}^{(\rho)} \cdot \mathbf{A} \cdot\left(\widehat{\mathbf{T}}_{N}^{(\rho)}\right)^{-1}, \\
& \mathbf{A}=\left(\widehat{\mathbf{T}}_{N}^{(\rho)}\right)^{-1} \cdot \mathbf{B} \cdot \widehat{\mathbf{T}}_{N}^{(\rho)} .
\end{aligned}
$$

The adopted compression scheme is described as follows [59]: (i) the image is divided into disjoint sub-blocks $\mathbf{A}_{j}$ of size $N \times N$; (ii) each sub-block is submitted to a selected transform $\widehat{\mathbf{T}}_{N}$ according to (12); (iii) using the standard zig-zag sequence [59], only the initial $r$ coefficients in each sub-block $\mathbf{B}_{j}$ are retained and the remaining $N^{2}-r$ coefficients are zeroed, resulting in sub-block $\overline{\mathbf{B}}_{j}$; (iv) the two-dimensional inverse transform is applied according (13), and (v) the reconstructed sub-blocks $\overline{\mathbf{A}}_{j}$ are adequately rearranged. The final reconstructed image $\overline{\mathbf{A}}$ is compared with the original image $\mathbf{A}$ for assessing the performance of $\widehat{\mathbf{T}}_{N}$. We adopted $N \in\{4,8,16,32\}$ and used the peak signal-to-noise ratio (PSNR) [60], and the mean 

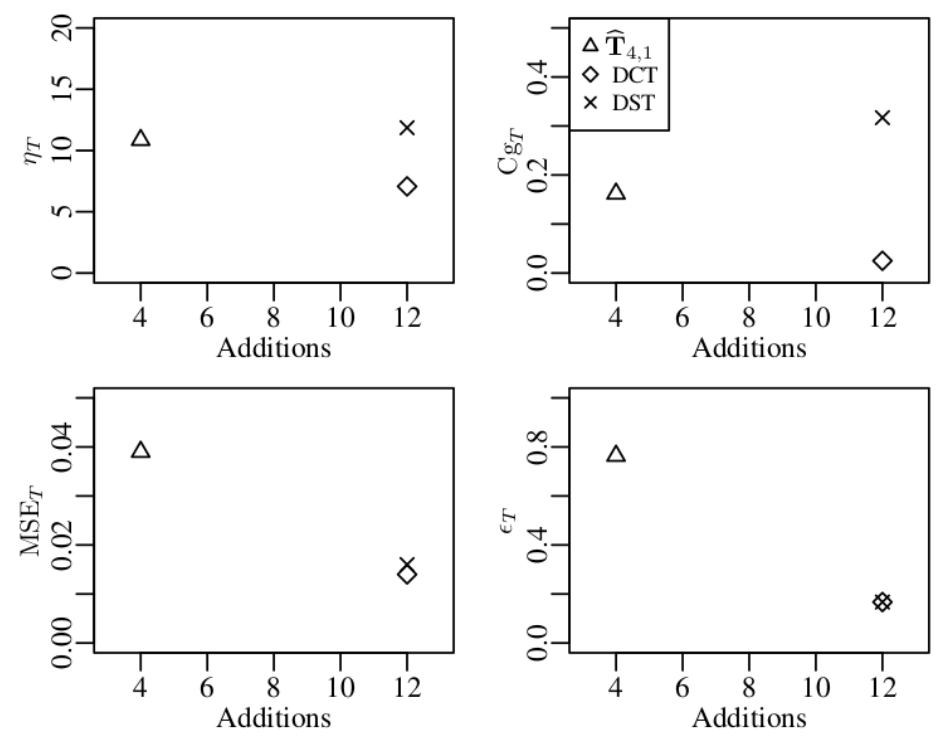

Figure 1: Arithmetic complexity versus the proposed figures of merit for each transform for $N=4$.

structural similarity index (MSSIM) [61] as figures of merit for image quality evaluation. The results were taken individually for 45 $512 \times 512$ 8-bit greyscale images obtained from [62] and averaged. For each transform length, we considered two approaches: (i) a qualitative analysis, based on the compressed Lena image with approximately $85 \%$ compression and (ii) a quantitative one, varying the value of $r$ for compression, considering the average MSSIM and PSNR values of the compressed images.

Figure 5 shows the original Lena image. The reconstructed images using the proposed transforms, DCT, and DST for $N=4,8,16$, and 32 are presented, respectively, in Figures 6, 7, 8, and 9 The corresponding compression ratio (CR) was $\mathrm{CR}=81.25 \%, 84.38 \%$, $84.38 \%, 84.86 \%$, respectively. Visually, the reconstructed images after compression exhibit quality comparable with the original image.

Figure 10 presents the average image quality measurements for different values of $N$ considering different levels of compression, comparing with the exact KLT for $\rho=0.1,0.6$, and 0.9 and the DCT. Figure 10(b) shows that the average MSSIM of $\widehat{\mathbf{T}}_{4,1}$ is very close to the results from the KLT and DCT. Figure 10(a) also shows that the values of the average PSNR of $\widehat{\mathbf{T}}_{4,1}$ are close to the values obtained by KLT and DCT. We can also notice that the approximation $\widehat{\mathbf{T}}_{4,1}$ presents average MSSIM values better than KLT itself when we retain fewer coefficients, $r$ ranging from zero to six. For $N=8$, one can see that $\widehat{\mathbf{T}}_{8,1}$ and $\widehat{\mathbf{T}}_{8,2}$ behave in a similar way according to the image quality measures. For $N=16, \widehat{\mathbf{T}}_{16,3}$ transform has considerably closer values to the DCT and KLT than the other approximations. This may be related to the fact that $\widehat{\mathbf{T}}_{16,3}$ is the obtained transform with a higher value of $\rho(\rho \geq 0.9)$. One can see that, for $r<10$, the approximation $\widehat{\mathbf{T}}_{16,3}$ presents better average MSSIM values than KLT itself. Figures 10(g) and 10(h) present the image quality measurements for $N=32$, that poses $\widehat{\mathbf{T}}_{32,4}$ as the best-performing approximate KLT for compression.

\subsection{Video coding}

In order to demonstrate the suitability of the introduced SKLTs in the video coding context, we embed the proposed approximations into a public available HEVC reference software [58]. The HEVC employs an integer DCT (IDCT) of lengths 4, 8, 16, and 32 [63], unlike its predecessors [64 67]. According to [22], the larger transforms generally work better for smooth image regions, whereas the textured areas are better handled by the small sized transforms.

For our experiment, we substituted the original set of IDCTs natively defined in the HEVC standard by our proposed KLT approximations. The original integer DST-VI [68] of length 4, responsible for residual coding in HEVC, is kept unchanged in the reference software. The main reason is that the optimal SKLT for length 4 is unique for all $\rho$ values (confer Table 1 , and it approaches the coding capabilities of the DCT [39]. We separated four suits of approximations, relating to the optimality in Equation (11): (i) $\widehat{\mathbf{T}}_{4,1}, \widehat{\mathbf{T}}_{8,1}$, $\widehat{\mathbf{T}}_{16,2}$, and $\widehat{\mathbf{T}}_{32,2}$ (Group I); (ii) $\widehat{\mathbf{T}}_{4,1}, \widehat{\mathbf{T}}_{8,1}, \widehat{\mathbf{T}}_{16,1}$, and $\widehat{\mathbf{T}}_{32,3}$ (Group II); (iii) $\widehat{\mathbf{T}}_{4,1}, \widehat{\mathbf{T}}_{8,2}, \widehat{\mathbf{T}}_{16,3}$, and $\widehat{\mathbf{T}}_{32,1}$ (Group III); and (iv) $\widehat{\mathbf{T}}_{4,1}, \widehat{\mathbf{T}}_{8,1}$, $\widehat{\mathbf{T}}_{16,3}$, and $\widehat{\mathbf{T}}_{32,4}$ (Group IV). Namely, Groups I, II, III, and IV are optimal regarding total MSE, total error energy, total unified coding gain, and total transform efficiency, respectively. Therefore, we substituted the original IDCT by each SKLT Group I-IV in the HEVC reference software.

In our experiments, we encoded the first 100 frames of one video sequence of each $\mathrm{A}$ to $\mathrm{F}$ class in accordance with the Common Test Conditions (CTC) document [69]. The considered 8bit standard video sequences were: PeopleOnStreet $(2560 \times 1600$ at $30 \mathrm{fps})$, BasketballDrive $(1920 \times 1080$ at $50 \mathrm{fps})$, RaceHorses $(832 \times 480$ at $30 \mathrm{fps})$, BlowingBubbles $(416 \times 240$ at $50 \mathrm{fps})$, KristenAndSara $(1280 \times 720$ at $60 \mathrm{fps})$, and BasketbalDrillText $(832 \times 480$ at $50 \mathrm{fps})$. We further considered the Foreman $(352 \times 288$ at $30 \mathrm{fps}$ [70], a standard 8-bit CIF video sequence adopted in related works like [31,33]. As done in [30], we set all the test parameters in accordance with the CTC documentation. Also, we considered the four standard 8-bit coding configurations in the Main profile: All Intra (AI), Random Access (RA) and Low-Delay B and P (LD-B and LD-P). We selected the frame-wise PSNR for each YUV color channel [63] as figure of merit. Then, for each video sequence, we computed the rate distortion (RD) curve considering quantization parameter (QP) values equal to $22,27,32$, and 37 [69].

Moreover, we measured the Bjøntegaard's delta PSNR (BDPSNR) [71 72] for the modified versions of the HEVC software. The average results per video for all the transform groups and coding 

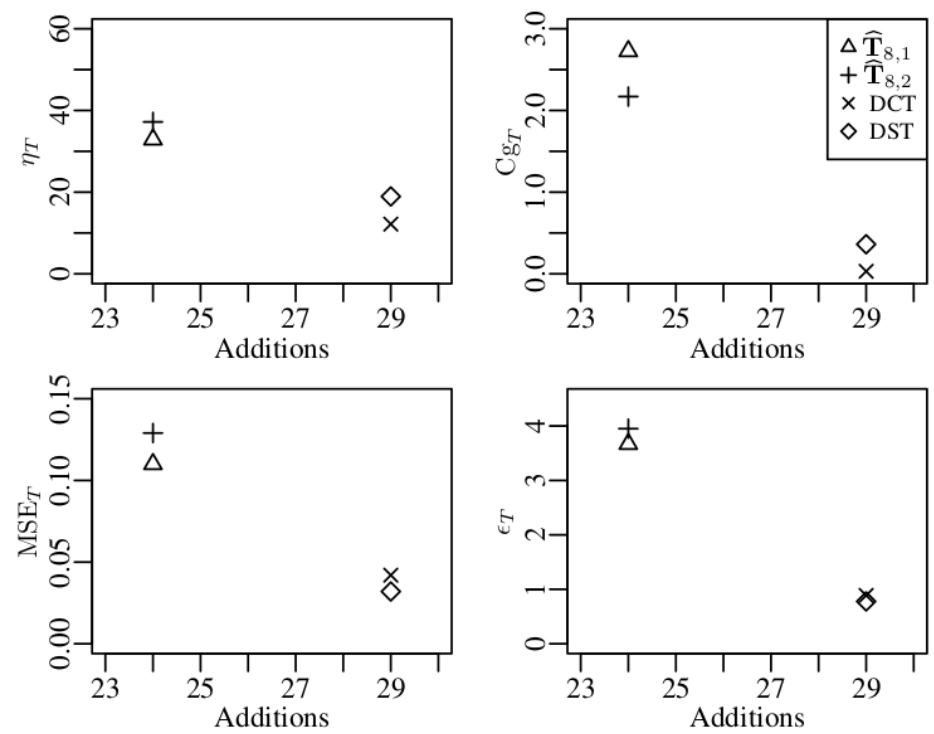

Figure 2: Arithmetic complexity versus the proposed figures of merit for each transform for $N=8$.

configurations are presented in Table 4 One can note from Table 4 that the Group IV performed better than the Group III on average. The metrics used for selecting both SKLT Groups III and IV maximize the coding efficiency of the transforms. From the table, Group IV outperformed Groups I and II in most of the cases regardless the configuration mode. This information can be confirmed in Figures 11, 12, 13, and 14, which show the RD curves for the four groups of transforms in $\mathrm{AI}, \mathrm{RA}, \mathrm{LD}-\mathrm{B}$, and LD-P configurations.

One can notice that the group of transforms that optimize the total transform efficiency metric (Group IV) tended to outperform the other three groups (Groups I, II, and III). The results in video coding corroborate those of the still-image experiments presented in Section 4.1

As a qualitative example, we present in Figure 15 the tenth frame of the KristenAndSara video encoded according to the default HEVC IDCT and the transforms in Groups I-IV in AI configuration. blue The presented metrics are a representation from the performance obtained by the proposed transforms on the other video sequences as well. Here, QP value was set to 32. Blocking artifacts are not easily perceptible, highlighting the applicability of the proposed SKLT.

\section{Conclusions}

Based on the signum function, we proposed a class of lowcomplexity KLT approximations, which are suitable for data decorrelation. These transforms are deterministically defined for processing data at a wide range correlation, which differs from the fast approximations for the KLT already known in the literature. Since the proposed transforms are the first approximations for the KLT following this approach, there is nothing to compare with. In principle, all approximations derived from this method are novelty. In particular, we explicitly derived new transforms of length 4, 8, 16, and 32 and submitted them to a comprehensive assessment in the context of image and video coding. Total figures of merit were proposed for the selection of optimal transforms. The proposed approximations were tailored to decorrelate Markovian first-order data, at a very low arithmetic complexity and multiplierless operation. Besides the lower complexity, we also derived fast algorithms for the proposed transforms that were capable of reducing, even more, the arithmetic cost of its implementation. The proposed SKLT showed good compaction energy properties at a very low cost. Still image and video experiments demonstrate the suitability of the proposed approximations for image/video encoding, being capable of generating high quality images according to coding and similarity metrics. For future works, we wish to consider larger blocklenghts transforms and other applications for fully exploring the potential of the proposed transforms, such as the versatile video coding (VVC) standard [73-75], for example.

\section{A Matrix Factorization}

A.1 $\quad N=16$

For $N=16$, we can rewrite $\widehat{\mathbf{T}}_{16,1}, \widehat{\mathbf{T}}_{16,2}$, and $\widehat{\mathbf{T}}_{16,3}[39]$ as follows:

$$
\begin{aligned}
& \widehat{\mathbf{T}}_{16,1}=\frac{1}{4} \cdot \mathbf{P}_{16} \cdot \mathbf{A}_{16,3}^{\prime} \cdot \mathbf{A}_{16,2} \cdot \mathbf{A}_{16,1}, \\
& \widehat{\mathbf{T}}_{16,2}=\frac{1}{4} \cdot \mathbf{P}_{16} \cdot \mathbf{A}_{16,5}^{\prime} \cdot \mathbf{A}_{16,4}^{\prime} \cdot \mathbf{A}_{16,3}^{\prime \prime} \cdot \mathbf{A}_{16,2} \cdot \mathbf{A}_{16,1}, \\
& \widehat{\mathbf{T}}_{16,3}=\frac{1}{4} \cdot \mathbf{P}_{16} \cdot \mathbf{A}_{16,5}^{\prime \prime} \cdot \mathbf{A}_{16,4}^{\prime \prime} \cdot \mathbf{A}_{16,3}^{\prime \prime \prime} \cdot \mathbf{A}_{16,2} \cdot \mathbf{A}_{16,1},
\end{aligned}
$$

where

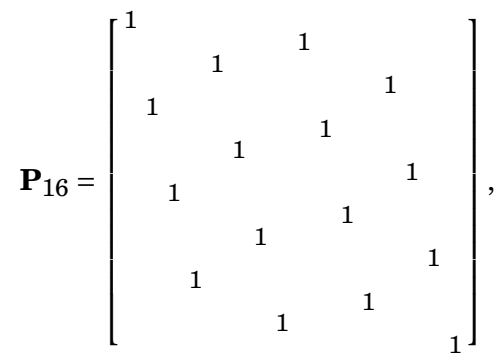

$$
\begin{aligned}
& \mathbf{A}_{16,1}=\left[\begin{array}{cc}
\mathbf{I}_{8} & \overline{\mathbf{I}}_{8} \\
\overline{\mathbf{I}}_{8} & -\mathbf{I}_{8}
\end{array}\right], \quad \mathbf{A}_{16,2}=\left[\begin{array}{ll}
\mathbf{A}_{8,1} & \\
& \mathbf{A}_{8,1}
\end{array}\right],
\end{aligned}
$$



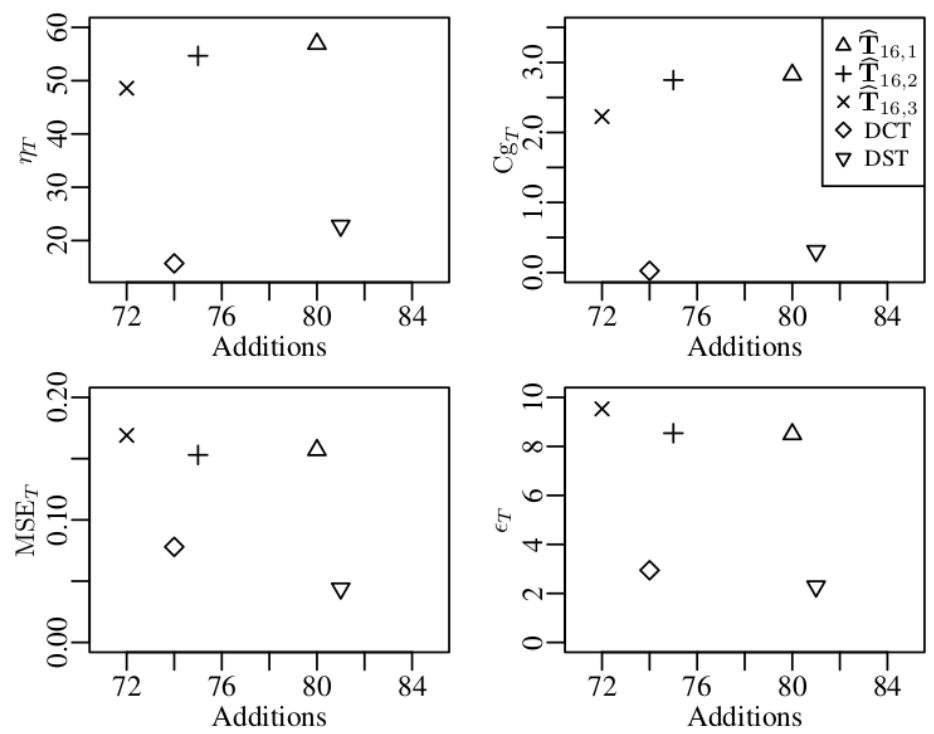

Figure 3: Arithmetic complexity versus the proposed figures of merit for each transform for $N=16$.

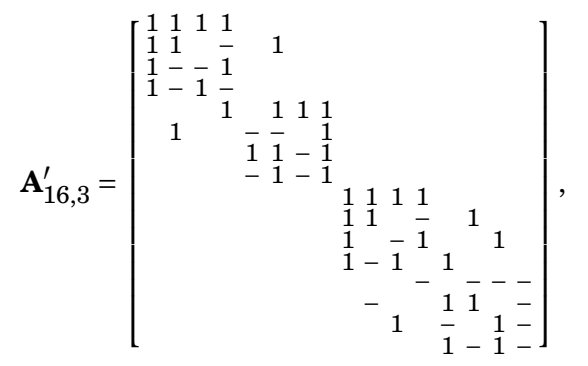

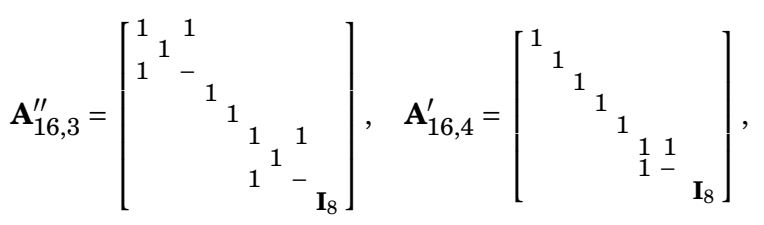

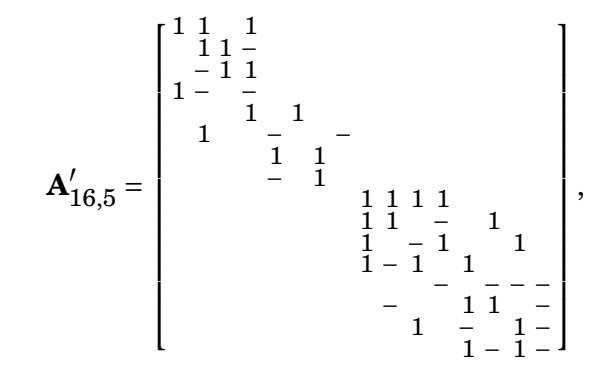

$$
\begin{aligned}
& \mathbf{A}_{16,3}^{\prime \prime \prime}=\left[\begin{array}{llll}
\mathbf{A}_{8,2} & & \\
& \mathbf{I}_{8}
\end{array}\right], \quad \mathbf{A}_{16,4}^{\prime \prime}=\left[\begin{array}{lllllll}
1 & 1 & & & & & \\
& & & & & & \\
& 1 & 1 & & & \\
& & 1 & - & & & \\
& & & & & & \\
& & & 1 & & \\
& & & & 1 & \\
& & & & & & \\
& & & & & \mathbf{I}_{8}
\end{array}\right],
\end{aligned}
$$

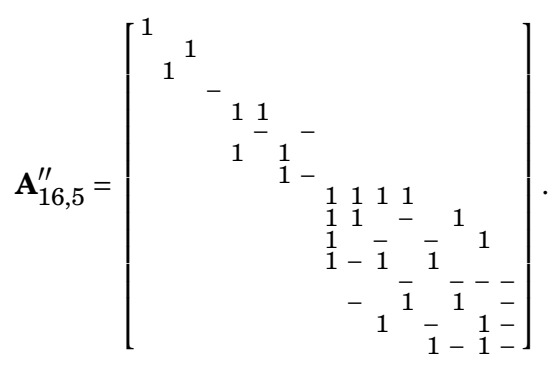$$
\text { A.2 } N=32
$$$$
\text { Considering } N=32 \text {, we have: }
$$$$
\begin{aligned}
& \widehat{\mathbf{T}}_{32,1}=\frac{1}{\sqrt{32}} \cdot \mathbf{P}_{32} \cdot \mathbf{A}_{32,3}^{\prime} \cdot \mathbf{A}_{32,2} \cdot \mathbf{A}_{32,1} \\
& \widehat{\mathbf{T}}_{32,2}=\frac{1}{\sqrt{32}} \cdot \mathbf{P}_{32} \cdot \mathbf{A}_{32,3}^{\prime \prime} \cdot \mathbf{A}_{32,2} \cdot \mathbf{A}_{32,1} \\
& \widehat{\mathbf{T}}_{32,3}=\frac{1}{\sqrt{32}} \cdot \mathbf{P}_{32} \cdot \mathbf{A}_{32,3}^{\prime \prime \prime} \cdot \mathbf{A}_{32,2} \cdot \mathbf{A}_{32,1} \\
& \widehat{\mathbf{T}}_{32,4}=\frac{1}{\sqrt{32}} \cdot \mathbf{P}_{32} \cdot \mathbf{A}_{32,6}^{\prime} \cdot \mathbf{A}_{32,5}^{\prime} \cdot \mathbf{A}_{32,4}^{\prime} \cdot \mathbf{A}_{32,3}^{\prime \prime \prime} \cdot \mathbf{A}_{32,2} \cdot \mathbf{A}_{32,1}
\end{aligned}
$$ 

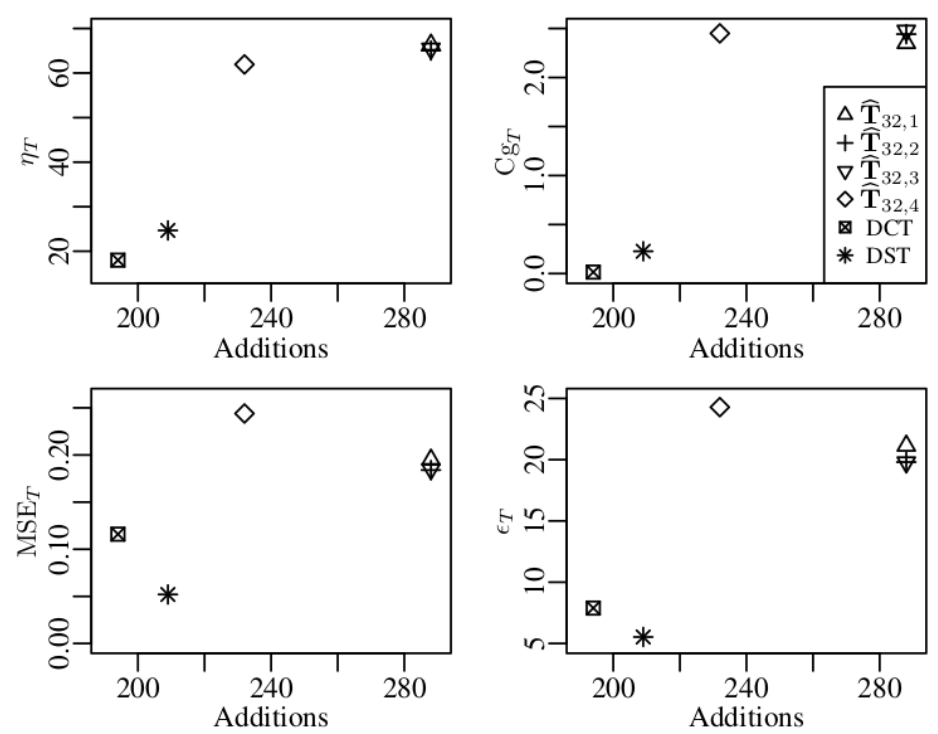

Figure 4: Arithmetic complexity versus the proposed figures of merit for each transform for $N=32$.

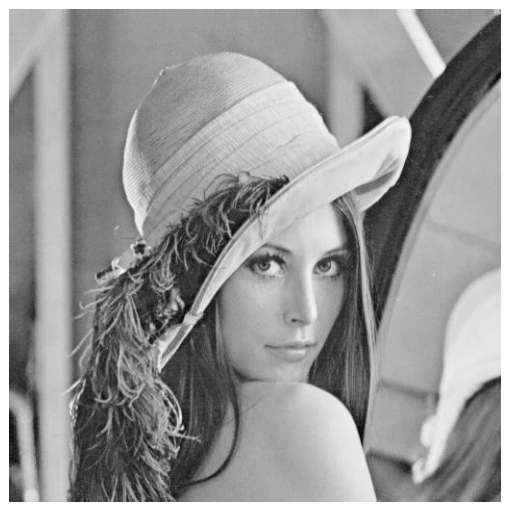

Figure 5: Original Lena image.

where

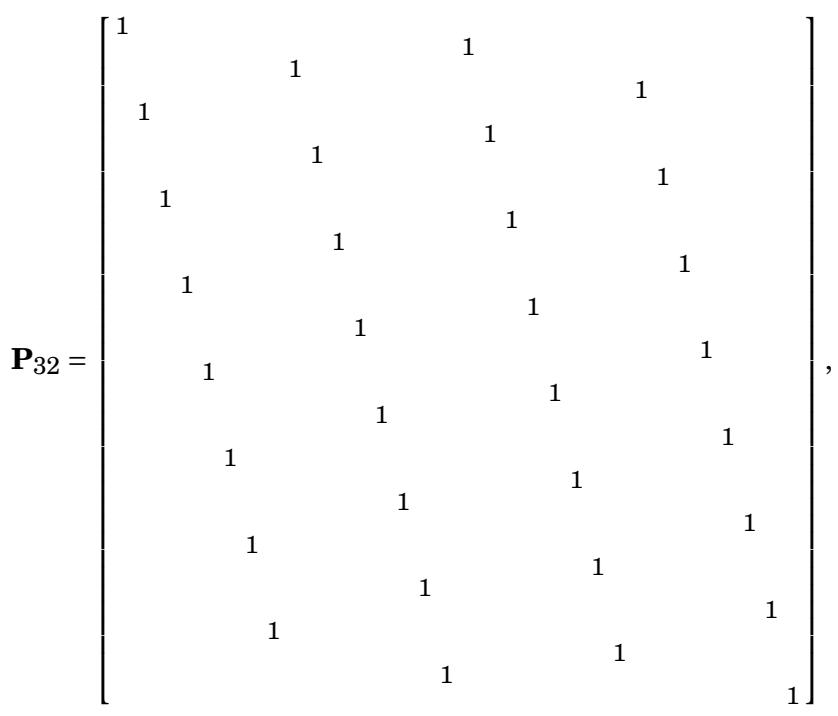

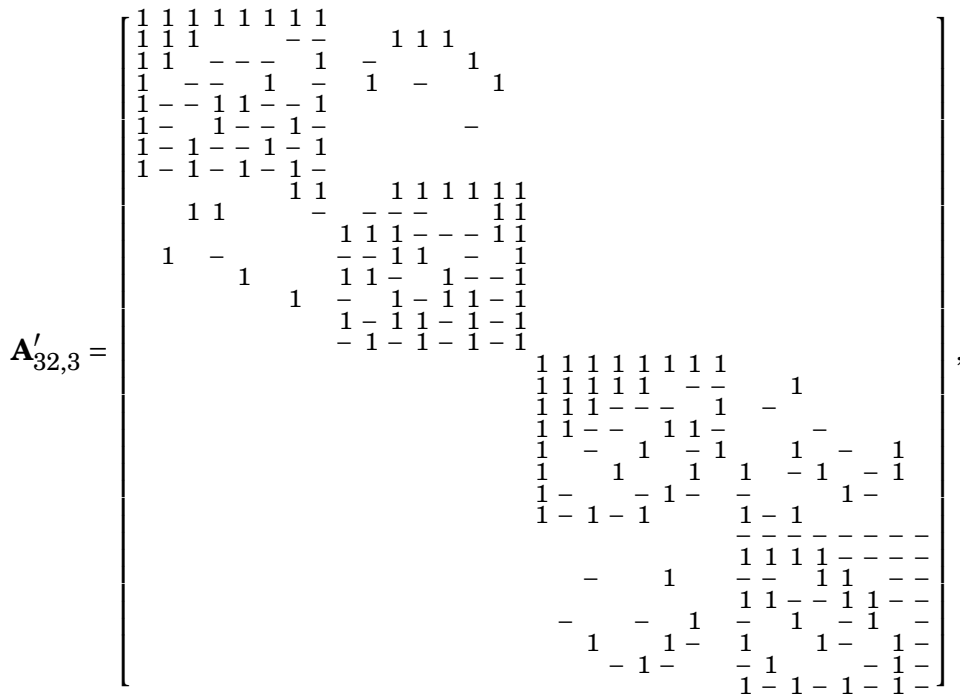

$\mathbf{A}_{32,1}=\left[\begin{array}{cc}\mathbf{I}_{16} & \overline{\mathbf{I}}_{16} \\ \overline{\mathbf{I}}_{16} & -\mathbf{I}_{16}\end{array}\right], \quad \mathbf{A}_{32,2}=\left[\begin{array}{cc}\mathbf{A}_{16,1} & \\ & \mathbf{A}_{16,1}\end{array}\right]$, 

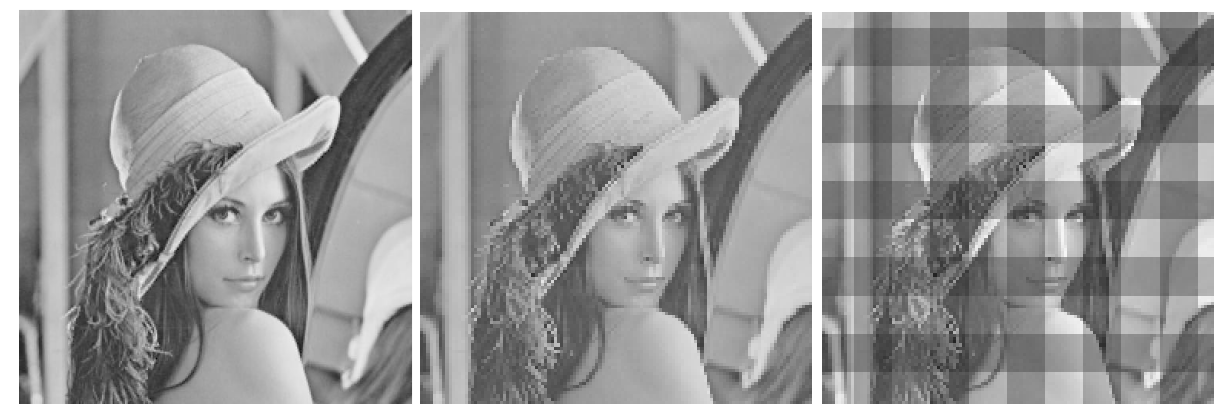

(a) $\widehat{T}_{4,1} 39, \operatorname{PSNR}=30.758, \operatorname{MSSIM}\left(\right.$ b) $\operatorname{DCT}_{4}(\rho \rightarrow 1), \operatorname{PSNR}=32.001,\left(\right.$ c) $\operatorname{DST}_{4}(\rho \rightarrow 0), \operatorname{PSNR}=15.491$ $=0.846 \quad$ MSSIM $=0.871 \quad$ MSSIM $=0.127$

Figure 6: Compressed Lena images for $N=4$ and $r=3$.
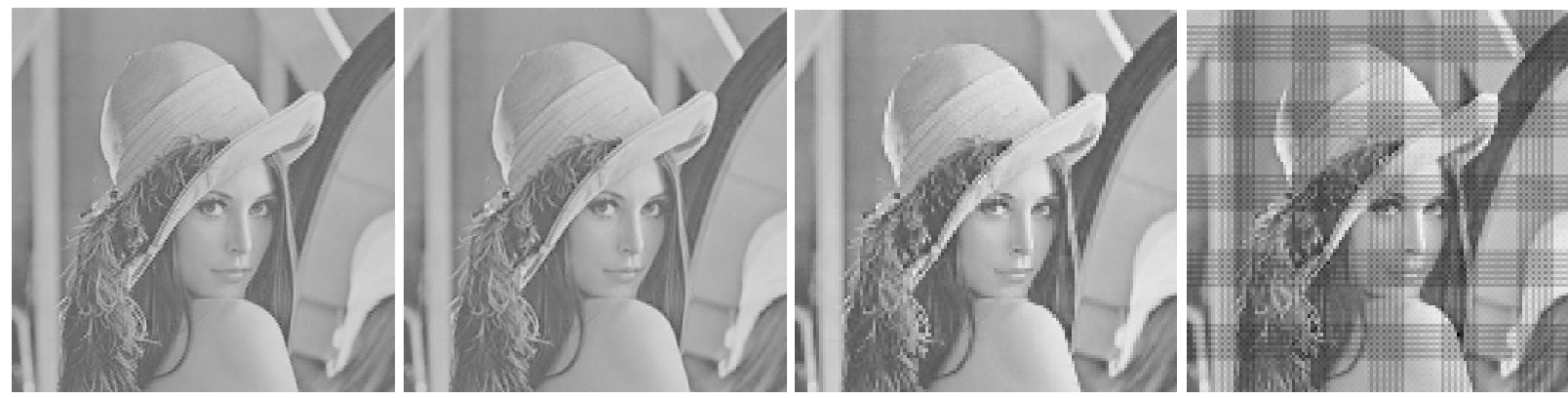

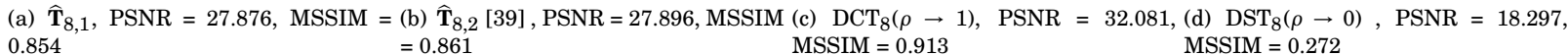

Figure 7: Compressed Lena images for $N=8$ and $r=10$.
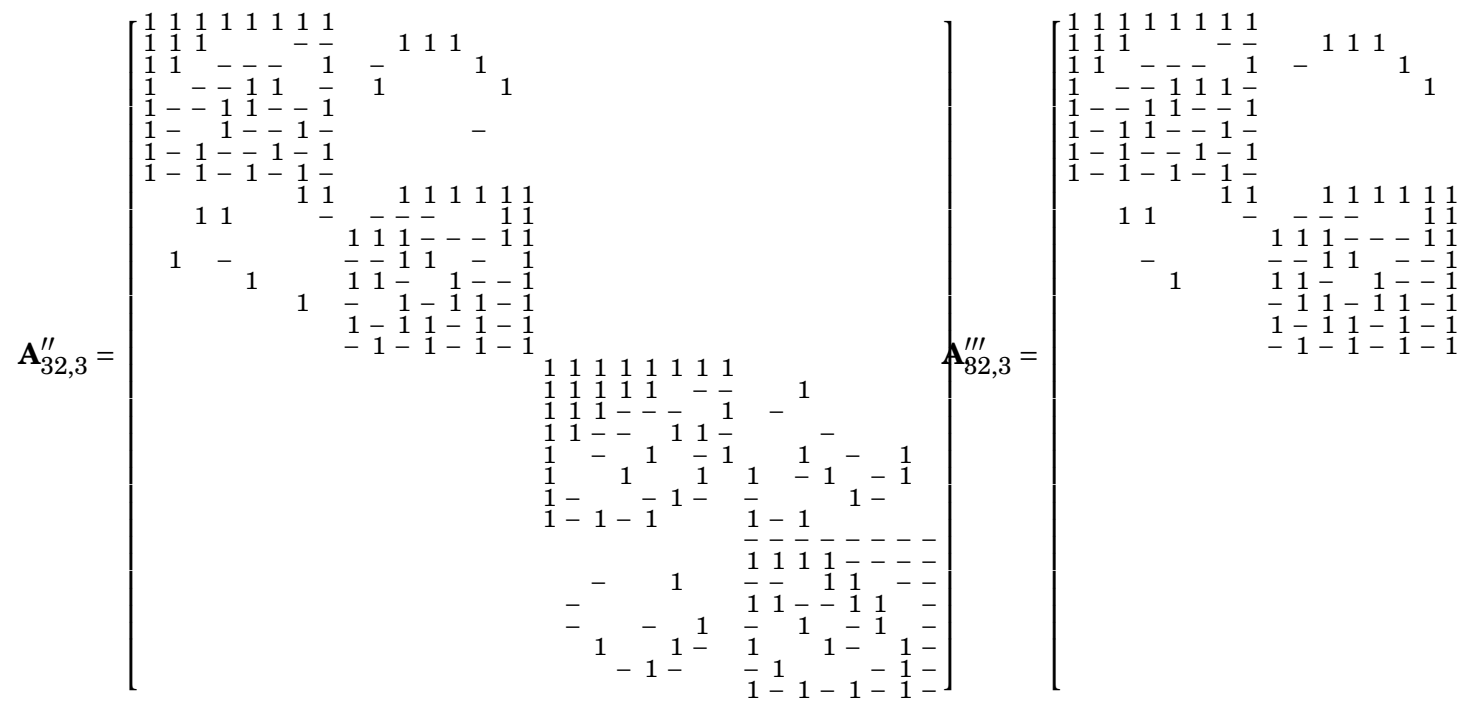

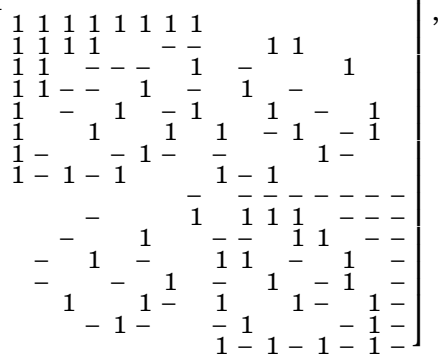

$$
\mathbf{A}_{32,3}^{\prime \prime \prime \prime}=\left[\begin{array}{ll}
\mathbf{A}_{16,2} & \\
& \mathbf{I}_{16}
\end{array}\right], \quad \mathbf{A}_{32,4}^{\prime}=\left[\begin{array}{ll}
\mathbf{A}_{16,3}^{\prime \prime \prime} & \\
& \mathbf{I}_{16}
\end{array}\right], \quad \mathbf{A}_{32,5}^{\prime}=\left[\begin{array}{ll}
\mathbf{A}_{16,4}^{\prime \prime} & \\
& \mathbf{I}_{16}
\end{array}\right]
$$



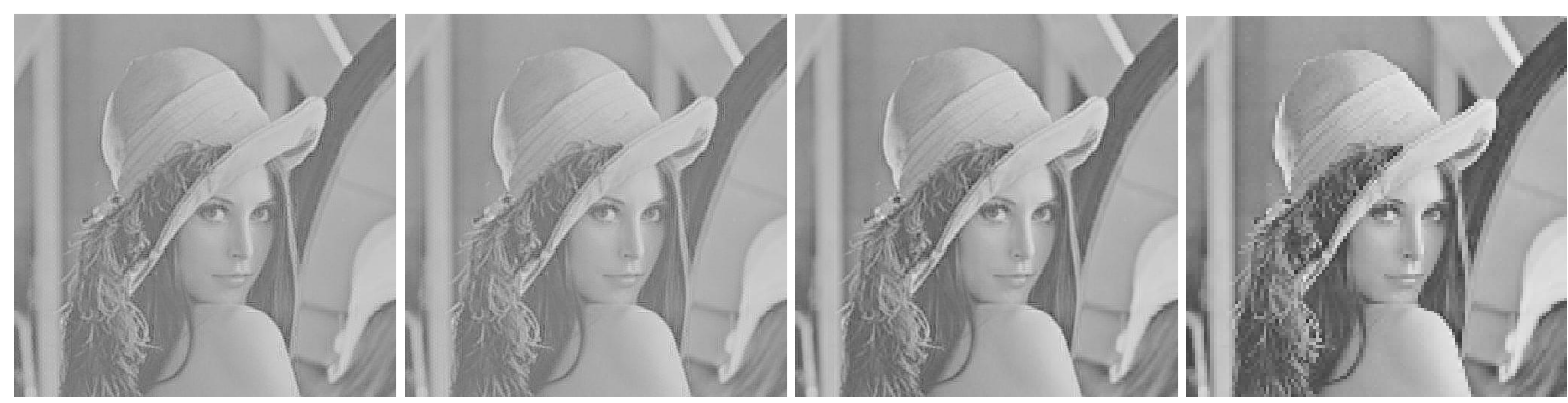

(a) $\widehat{\mathbf{T}}_{16,1}, \mathrm{PSNR}=25.717, \mathrm{MSSIM}=$ (b) $\widehat{\mathbf{T}}_{16,2}, \mathrm{PSNR}=25.887, \mathrm{MSSIM}=$ (c) $\widehat{\mathbf{T}}_{16,3} 39, \quad$ PSNR $=27.200$, (d) $\operatorname{DCT}_{16}(\rho \rightarrow 1), \operatorname{PSNR}=32.494$, 0.862 0.866 MSSIM $=0.892$

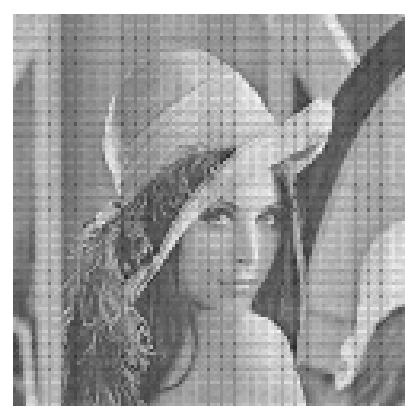

(e) $\operatorname{DST}_{16}(\rho \rightarrow 0)$, PSNR $=22.639$, MSSIM $=0.532$

Figure 8: Compressed Lena images for $N=16$ and $r=40$.

and

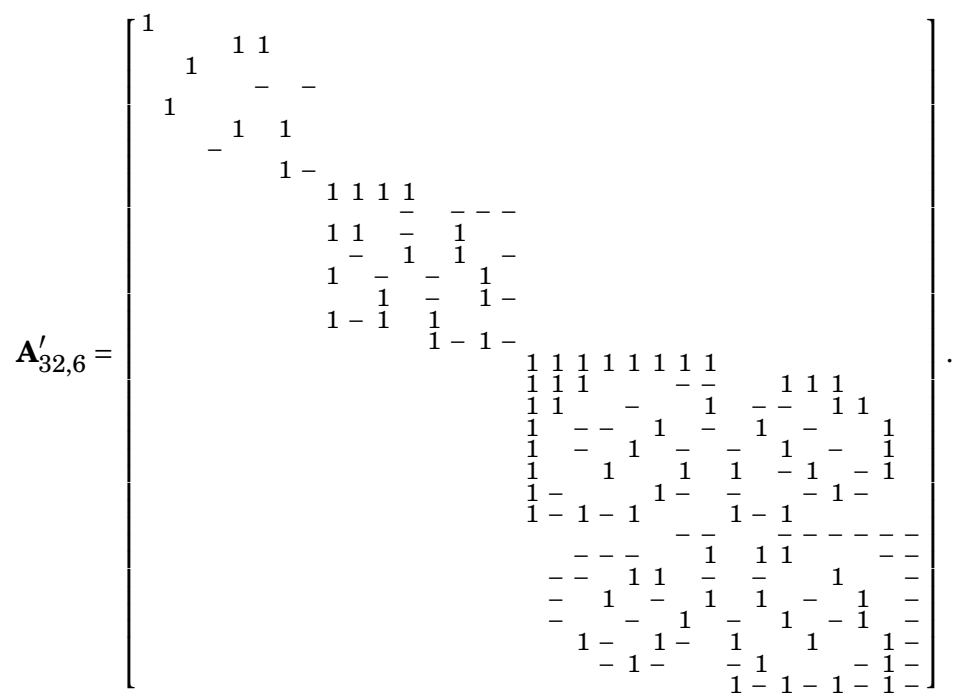

\section{Acknowledgments}

We gratefully acknowledge partial financial support from Coordenação de Aperfeiçoamento de Pessoal de Nível Superior (CAPES), Conselho Nacional de Desenvolvimento Científico e Tecnológico (CNPq) and Fundação de Amparo a Ciência e Tecnologia de Pernambuco (FACEPE), Brazil. 

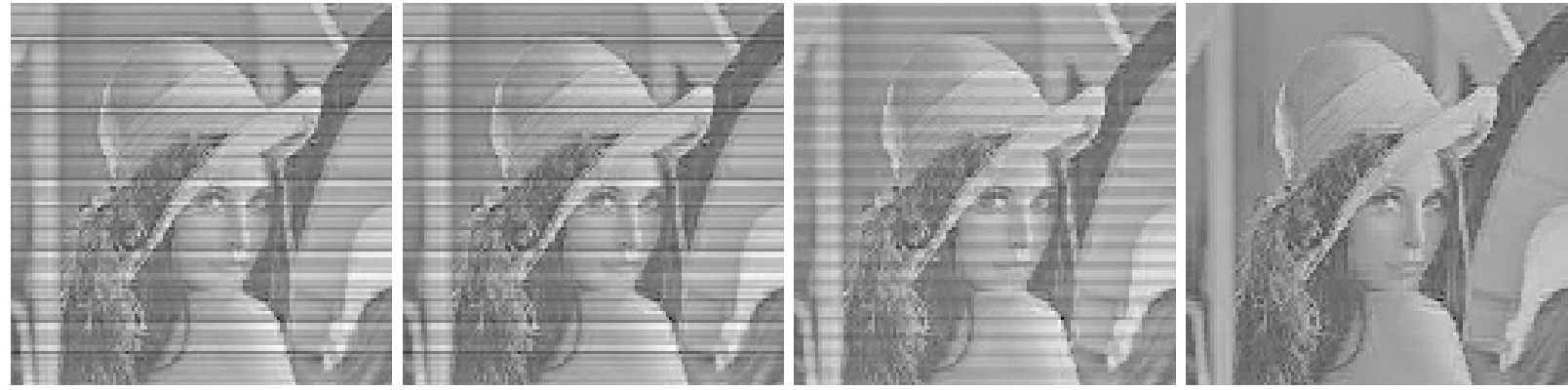

(a) $\widehat{\mathbf{T}}_{32,1}$, PSNR $=17.519$, MSSIM $=\left(\right.$ b) $\widehat{\mathbf{T}}_{32,2}$, PSNR $=17.573$, MSSIM $=\left(\right.$ c) $\widehat{\mathbf{T}}_{32,3}$, PSNR $=20.632$, MSSIM $=(\mathrm{d}) \widehat{\mathbf{T}}_{32,4}$, PSNR $=25.997$, MSSIM $=$ 0.473 0.476 0.600 0.911
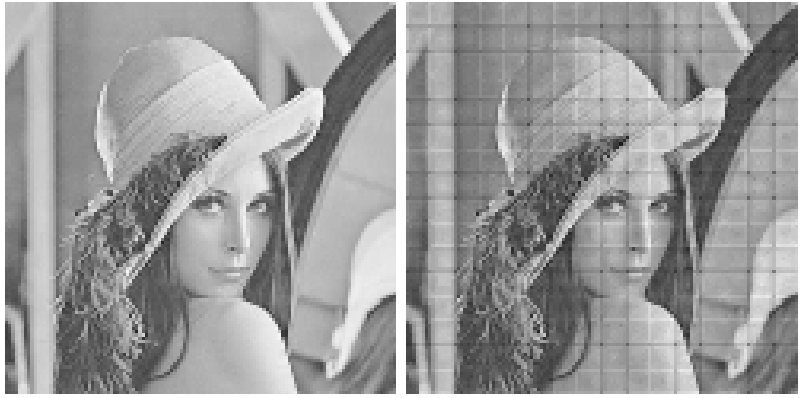

(e) $\operatorname{DCT}_{32}(\rho \rightarrow 1)$, PSNR $=32.996,($ f $) \operatorname{DST}_{32}(\rho \rightarrow 0)$, PSNR $=26.089$ MSSIM $=0.969$ MSSIM $=0.781$

Figure 9: Compressed Lena images for $N=32$ and $r=155$.

Table 4: Average BD-PSNR of the modified HEVC reference software for tested video sequences

\begin{tabular}{cccccc}
\hline \multirow{2}{*}{ Configuration } & Video sequence & \multicolumn{4}{c}{ Transforms } \\
\cline { 3 - 5 } & PeopleOnStreet & -0.6074 & -0.6127 & -0.5696 & -0.5774 \\
& BasketballDrive & -0.6194 & -0.6434 & -0.4549 & -0.4452 \\
AI & RaceHorses & -0.8221 & -0.8219 & -0.8280 & -0.8290 \\
& KristenAndSara & -0.3446 & -0.3464 & -0.3142 & -0.3361 \\
& BlowingBubbles & -0.7275 & -0.7425 & -0.5495 & -0.5391 \\
& BasketballDrillText & -0.3163 & -0.3217 & -0.2680 & -0.2844 \\
& Foreman & -0.3030 & -0.3075 & -0.2636 & -0.2794 \\
\hline \multirow{4}{*}{ RA } & PeopleOnStreet & -0.3995 & -0.4030 & -0.3748 & -0.3797 \\
& BasketballDrive & -0.4855 & -0.5054 & -0.3629 & -0.3423 \\
& RaceHorses & -1.2096 & -1.2150 & -1.1687 & -1.1435 \\
& BlowingBubbles & -0.2784 & -0.2849 & -0.2440 & -0.2525 \\
& BasketballDrillText & -0.4108 & -0.4095 & -0.3184 & -0.3241 \\
LD-B & -0.2532 & -0.2561 & -0.2127 & -0.2290 \\
\hline & Foreman & -0.4900 & -0.5089 & -0.3548 & -0.3117 \\
& RaceHetballDrive & -1.1279 & -1.1342 & -1.1324 & -1.1028 \\
& BlowingBubbles & -0.2978 & -0.3150 & -0.2519 & -0.2610 \\
& KristenAndSara & -0.4069 & -0.4245 & -0.2958 & -0.2740 \\
& BasketballDrillText & -0.4609 & -0.4629 & -0.3616 & -0.3528 \\
& Foreman & -0.3147 & -0.2988 & -0.2421 & -0.2436 \\
\hline \multirow{4}{*}{ LD-P } & BasketballDrive & -0.4932 & -0.5139 & -0.3542 & -0.3153 \\
& RaceHorses & -1.0903 & -1.0940 & -1.0929 & -1.0637 \\
& BlowingBubbles & -0.2905 & -0.2958 & -0.2438 & -0.2404 \\
& KristenAndSara & -0.3889 & -0.4062 & -0.2791 & -0.2544 \\
& BasketballDrillText & -0.4317 & -0.4369 & -0.3400 & -0.3361 \\
& Foreman & -0.2828 & -0.2927 & -0.2308 & -0.2381 \\
\hline & & & & &
\end{tabular}




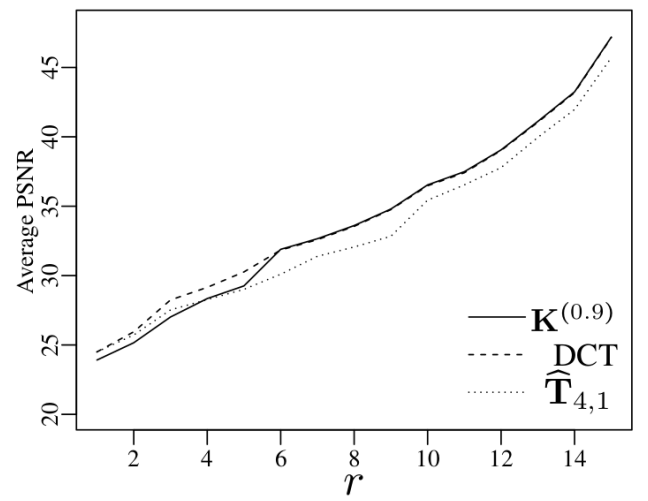

(a) Average PSNR $(N=4)$

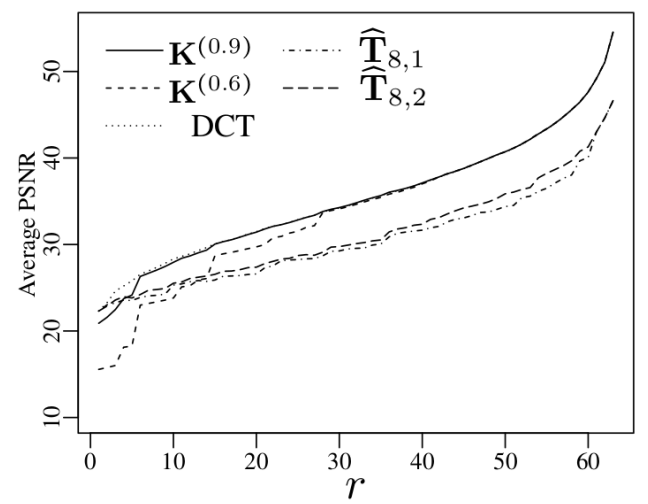

(c) Average PSNR $(N=8)$

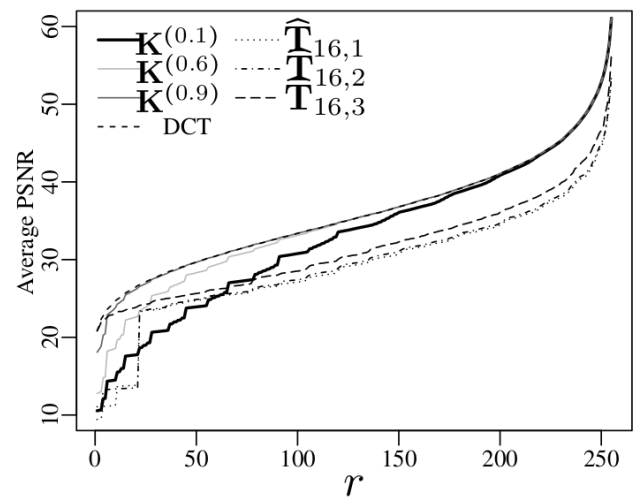

(e) Average PSNR $(N=16)$

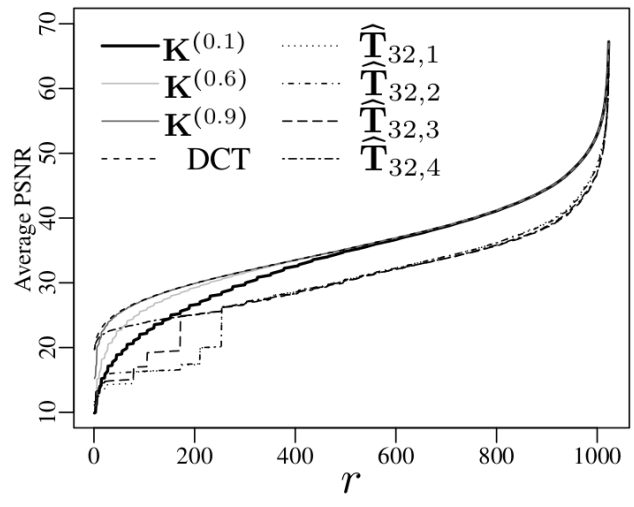

(g) Average PSNR $(N=32)$

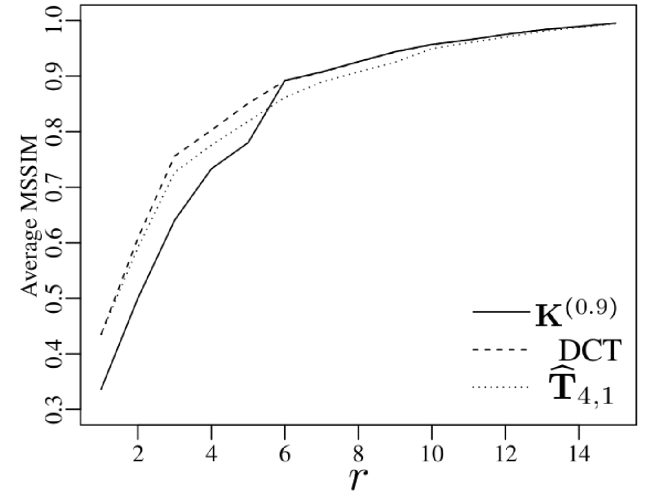

(b) Average MSSIM $(N=4)$

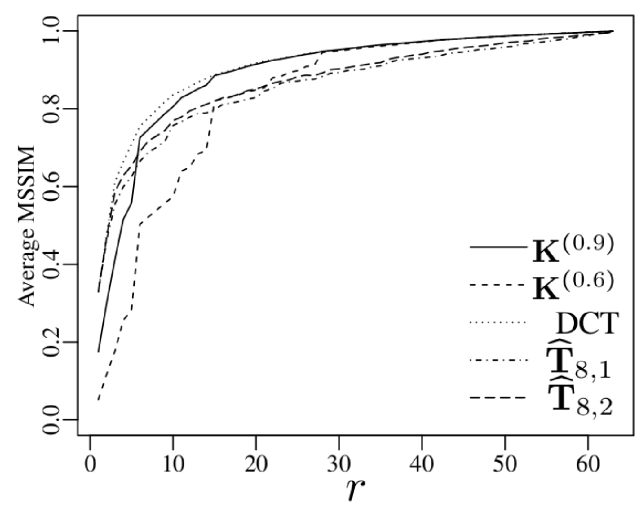

(d) Average MSSIM $(N=8)$

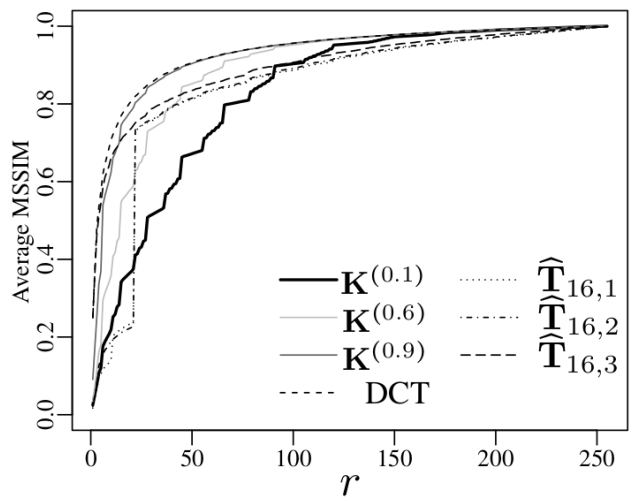

(f) Average $\operatorname{MSSIM}(N=16)$

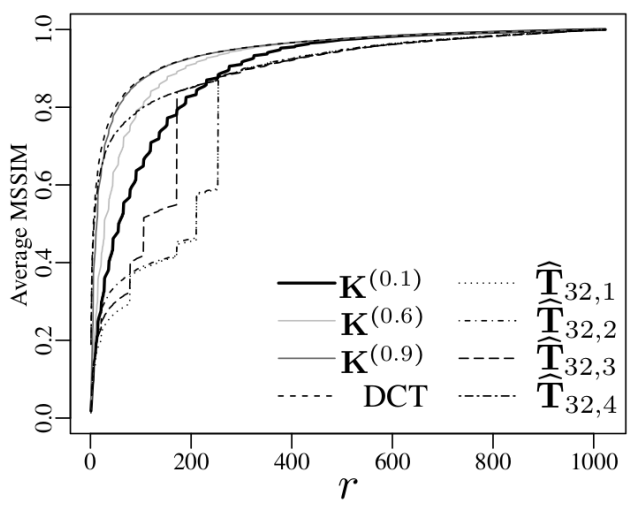

(h) Average MSSIM $(N=32)$

Figure 10: Image quality measurements for different levels of compression. 


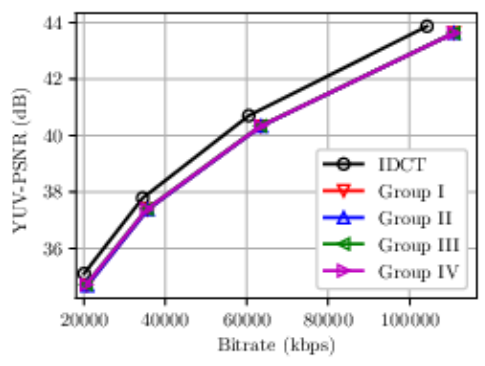

(a)

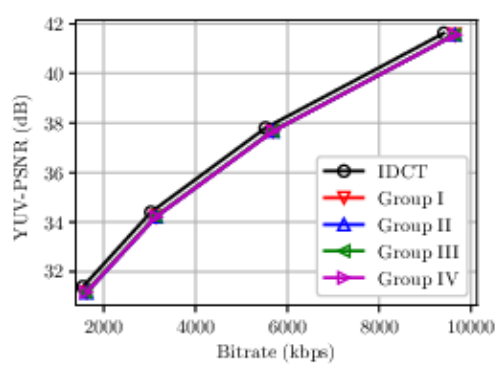

(d)

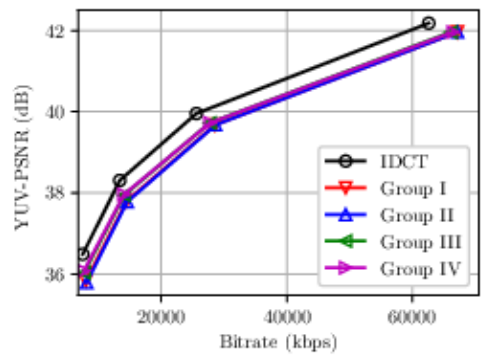

(b)

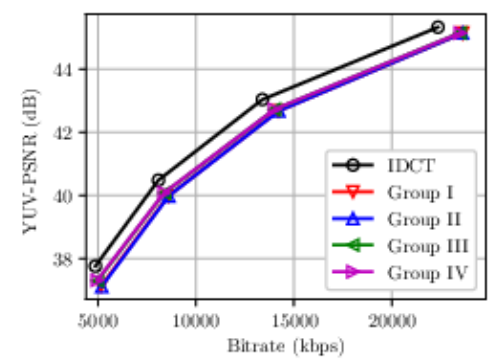

(e)

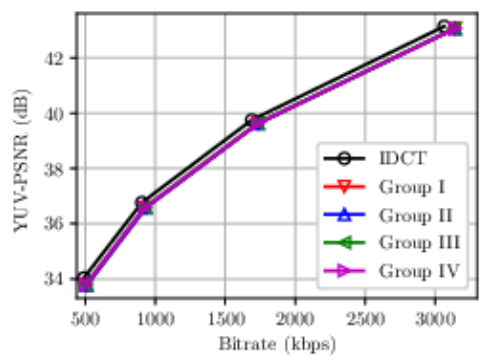

(g)

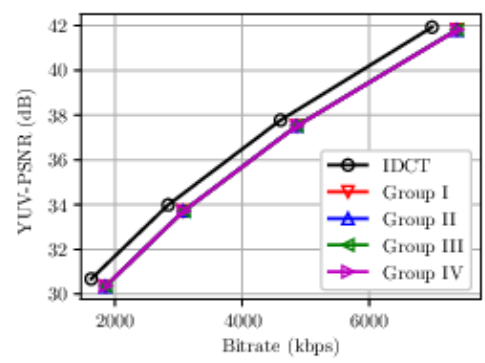

(c)

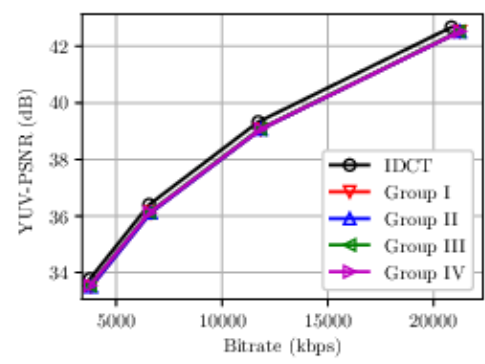

(f)

Figure 11: RD curves of the modified HEVC versions for test sequences in Main profile and AI configuration: (a) People0nStreet, (b) BasketballDrive, (c) RaceHorses, (d) BlowingBubbles, (e) KristenAndSara, (f) BasketbalDrillText, and (g) Foreman. 


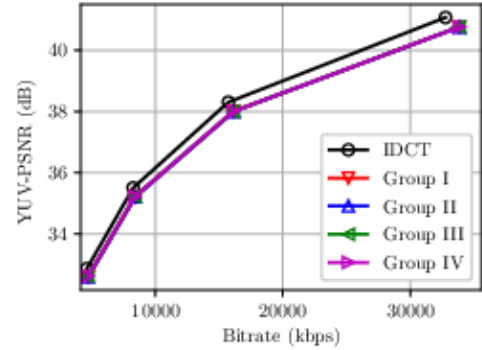

(a)

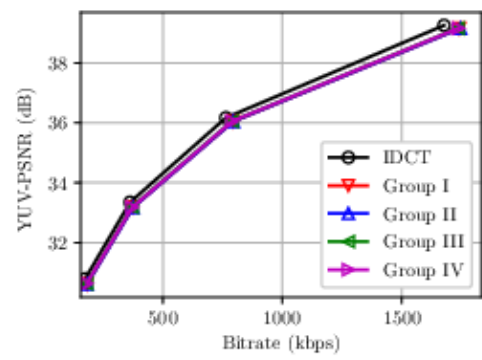

(d)

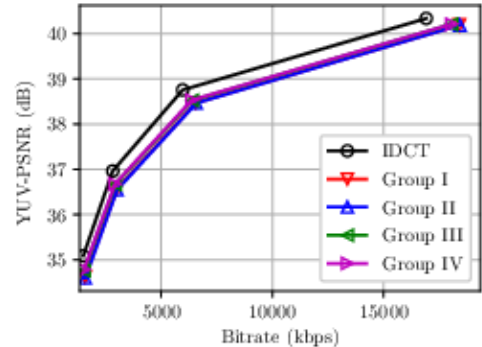

(b)

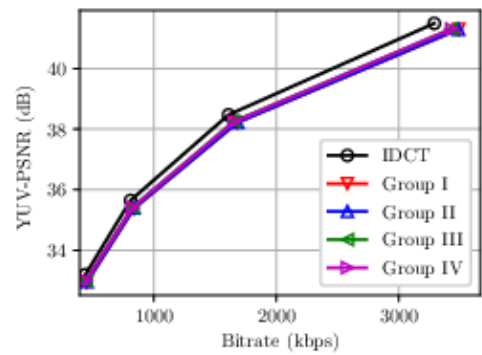

(e)

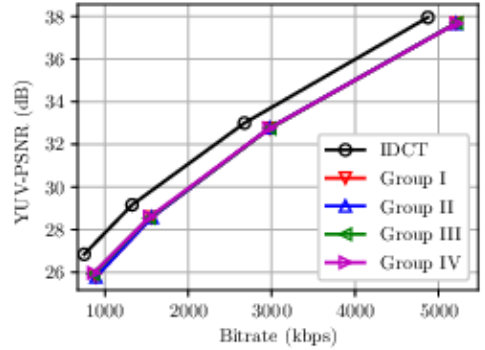

(c)

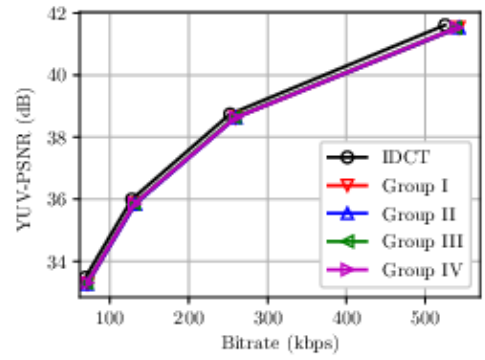

(f)

Figure 12: RD curves of the modified HEVC versions for test sequences in Main profile and RA configuration: (a) People0nStreet, (b) BasketballDrive, (c) RaceHorses, (d) BlowingBubbles, (e) BasketbalDrillText, and (f) Foreman.

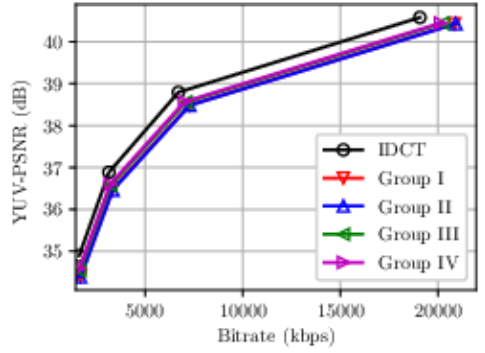

(a)

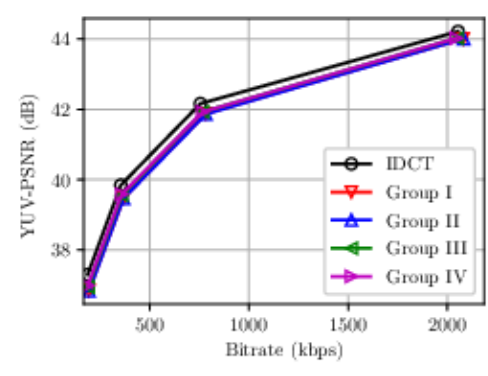

(d)

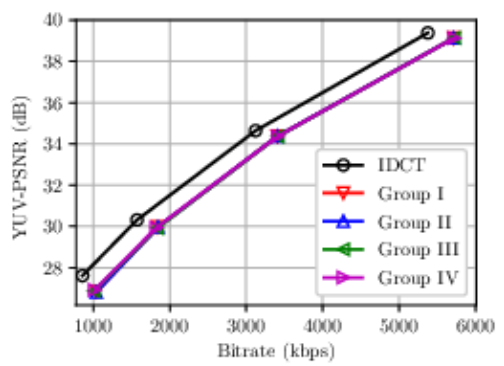

(b)

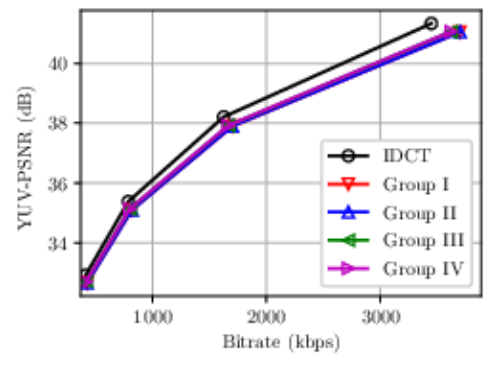

(e)

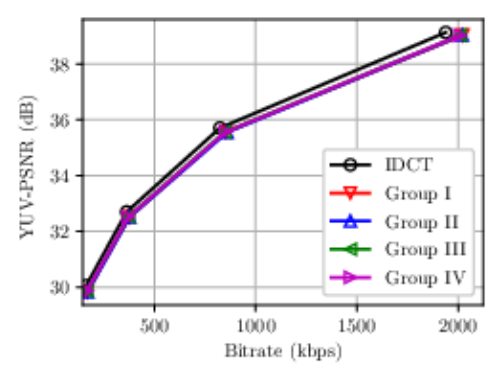

(c)

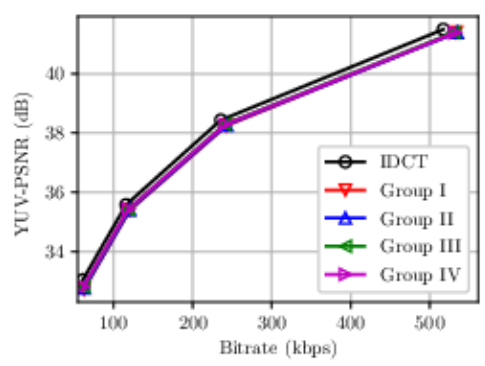

(f)

Figure 13: RD curves of the modified HEVC versions for test sequences in Main profile and LD-B configuration: (a) BasketballDrive, (b) RaceHorses, (c) BlowingBubbles, (d) KristenAndSara, (e) BasketbalDrillText, and (f) Foreman. 


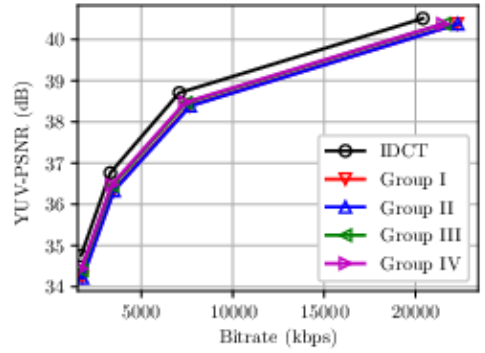

(a)

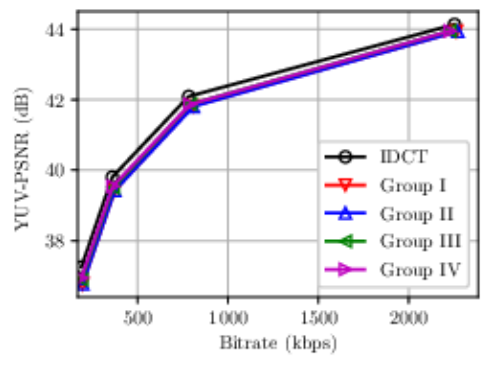

(d)

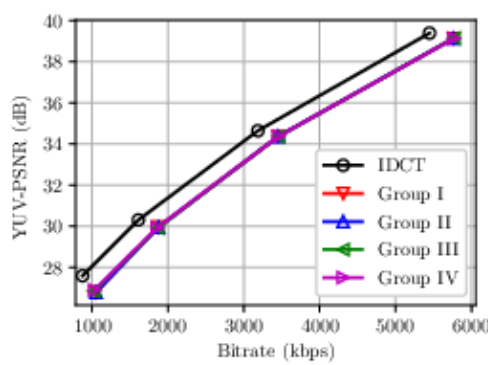

(b)

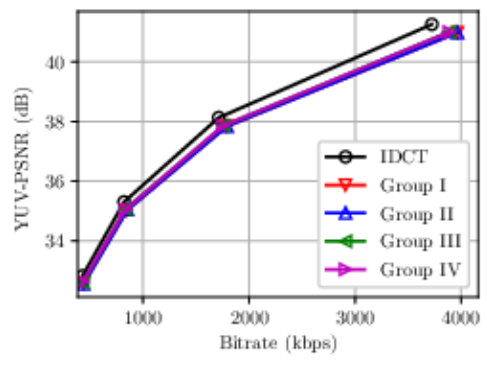

(e)

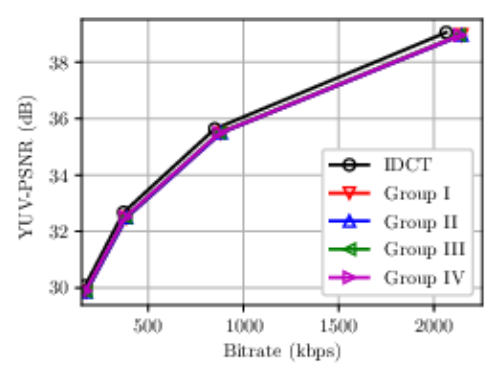

(c)

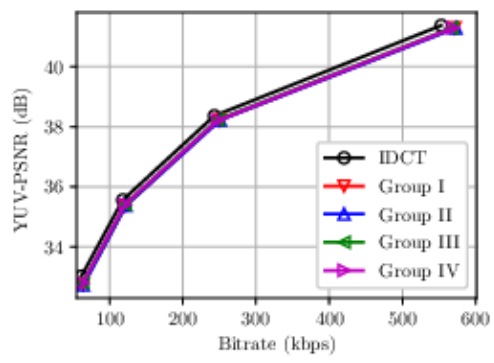

(f)

Figure 14: RD curves of the modified HEVC versions for test sequences in Main profile and LD-P configuration: (a) BasketballDrive, (b) RaceHorses, (c) BlowingBubbles, (d) KristenAndSara, (e) BasketbalDrillText, and (f) Foreman.
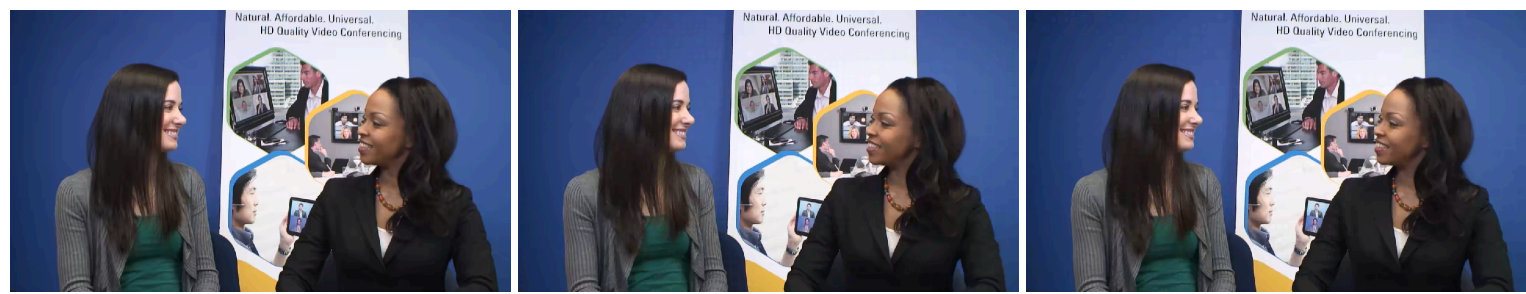

(a) PSNR-Y = 39.4857dB, PSNR-U = 43.5883dB (b) PSNR-Y = 39.0457dB, PSNR-U = 43.0431dB (c) PSNR-Y = 39.0571dB, PSNR-U = 43.0027dB and PSNR-V $=44.4961 \mathrm{~dB}$ and PSNR-V $=43.9074 \mathrm{~dB}$ and PSNR-V $=43.8623 \mathrm{~dB}$
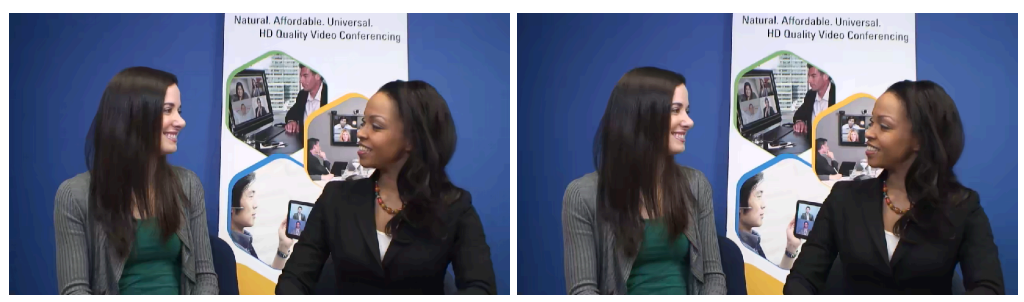

(d) PSNR-Y $=39.1155 \mathrm{~dB}$, PSNR-U $=43.1140 \mathrm{~dB}$ (e) PSNR-Y $=39.1320 \mathrm{~dB}$, PSNR-U $=43.1120 \mathrm{~dB}$ and PSNR-V $=44.0624 \mathrm{~dB}$ and PSNR-V $=44.0912 \mathrm{~dB}$

Figure 15: Compression of the tenth frame of KristenAndSara using the default and modified versions of the HEVC software in configured to the AI mode and QP = 32. Results for the IDCT are shown in (a), and for the SKLT Groups I to IV in (b) to (e), respectively. 


\section{References}

[1] V. Britanak, P. C. Yip, and K. R. Rao, Discrete cosine and sine transforms: general properties, fast algorithms and integer approximations. Academic Press, 2010.

[2] I. T. Jolliffe, Principal Component Analysis. Springer-Verlag New York, 1986.

[3] H. A. Chipman and H. Gu, "Interpretable dimension reduction," Journal of Applied Statistics, vol. 32, no. 9, p. 969-987, 2005.

[4] R. A. Johnson, D. W. Wichern, et al., Applied multivariate statistical analysis, vol. 5. Prentice Hall Upper Saddle River, NJ, 2002.

[5] Q. Du and J. E. Fowler, "Hyperspectral image compression using JPEG2000 and principal component analysis," IEEE Geoscience and Remote Sensing Letters, vol. 4, no. 2, p. 201-205, 2007.

[6] H. Ochoa-Dominguez and K. R. Rao, Discrete Cosine Transform. CRC Press, 2019.

[7] W. Ray and R. Driver, "Further decomposition of the Karhunen-Loève series representation of a stationary random process," IEEE Transactions on Information Theory, vol. 16, no. 6, p. 663-668, 1970.

[8] A. K. Jain, "A fast Karhunen-Loève transform for a class of random processes," IEEE Transactions on Communications, vol. 24, no. 9, p. 1023-1029, 1976.

[9] L.-S. Lan and I. S. Reed, "Fast approximate Karhunen-Loève transform with applications to digital image coding," in Visual Communications and Image Processing'93, vol. 2094, pp. 444-455, International Society for Optics and Photonics, 1993.

[10] L.-S. Lan and I. S. Reed, "An improved JPEG image coder using the adaptive fast approximate Karhunen-Loève transform (AKLT)," in Proceedings of ICSIPNN'94. International Conference on Speech, Image Processing and Neural Networks, pp. 160-163, IEEE, 1994.

[11] A. Pirooz and I. Reed, "A new approximate Karhunen-Loève transform for data compression," in Conference Record of Thirty-Second Asilomar Conference on Signals, Systems and Computers, vol. 2, pp. 1471-1475, IEEE, 1998.

[12] Q. Yanyun, Z. Nanning, L. Cuihua, and Y. Zejian, "Updating algorithm for extracting the basis of Karhunen-Loève transform in nonzero mean data," in Proceedings 7th International Conference on Signal Processing, 2004. Proceedings. ICSP'04. 2004., vol. 2, pp. 1403-1406, IEEE, 2004.

[13] M. Cagnazzo, L. Cicala, G. Poggi, and L. Verdoliva, "Low-complexity compression of multispectral images based on classified transform coding," Signal Processing: Image Communication, vol. 21, no. 10, pp. 850-861, 2006.

[14] J. Sole, P. Yin, Y. Zheng, and C. Gomila, "Joint sparsity-based optimization of a set of orthonormal 2-D separable block transforms," in 2009 16th IEEE International Conference on Image Processing (ICIP), pp. 9-12, IEEE, 2009.

[15] I. Blanes, J. Serra-Sagrista, M. W. Marcellin, and J. Bartrina-Rapesta, "Divide-and-conquer strategies for hyperspectral image processing: A review of their benefits and advantages," IEEE Signal Processing Magazine, vol. 29, no. 3, pp. 71-81, 2012.

[16] P. Hao and Q. Shi, "Reversible integer KLT for progressive-to-lossless compression of multiple component images," in Proceedings 2003 International Conference on Image Processing, vol. 1, pp. I-633, IEEE, 2003.

[17] Y. Wongsawat, S. Oraintara, and K. R. Rao, "Integer sub-optimal Karhunen-Loève transform for multi-channel lossless EEG compression," in 2006 14th European Signal Processing Conference, pp. 1-5, IEEE, 2006.

[18] N. Ahmed, T. Natarajan, and K. R. Rao, "Discrete cosine transfom," IEEE Transactions on Computers, vol. C-23, no. 1, p. 90-93, 1974.

[19] R. Clarke, "Relation between the Karhunen-Loève and cosine transforms," in IEE Proceedings F-Communications, Radar and Signal Processing, vol. 128, p. 359-360, IET, 1981.

[20] R. Clarke, "Relation between the karhunen-loeve and sine transforms," Electronics Letters, vol. 20, no. 1, pp. 12-13, 1984.

[21] G. K. Wallace, "The JPEG still picture compression standard," IEEE Transactions on Consumer Electronics, vol. 38, no. 1, p. xviii-xxxiv, 1992.

[22] M. T. Pourazad, C. Doutre, M. Azimi, and P. Nasiopoulos, "HEVC: The new gold standard for video compression: How does HEVC compare with H.264/AVC?," IEEE Consumer Electronics Magazine, vol. 1, no. 3, p. 36-46, 2012.

[23] R. J. Cintra, F. M. Bayer, and C. Tablada, "Low-complexity 8-point DCT approximations based on integer functions," Signal Processing, vol. 99, p. 201-214, 2014.

[24] R. J. Cintra, F. M. Bayer, Y. Pauchard, and A. Madanayake, "Low-complexity DCT approximations for biomedical signal processing in big data," in Signal Processing and Machine Learning for Biomedical Big Data, p. 151-176, CRC Press, 2018.

[25] S. Bouguezel, M. O. Ahmad, and M. Swamy, "Low-complexity $8 \times 8$ transform for image compression,” Electronics Letters, vol. 44, no. 21, p. 1249-1250, 2008.

[26] T. Sheltami, M. Musaddiq, and E. Shakshuki, "Data compression techniques in wireless sensor networks," Future Generation Computer Systems, vol. 64, pp. 151-162, 2016.

[27] U. S. Potluri, A. Madanayake, R. J. Cintra, F. M. Bayer, S. Kulasekera, and A. Edirisuriya, "Improved 8-point approximate DCT for image and video compression requiring only 14 additions," IEEE Transactions on Circuits and Systems I: Regular Papers, vol. 61, no. 6, p. 1727-1740, 2014.

[28] R. J. Cintra and F. M. Bayer, "A DCT approximation for image compression," IEEE Signal Processing Letters, vol. 18, no. 10, p. 579-582, 2011.

[29] S. Bouguezel, M. O. Ahmad, and M. Swamy, "Binary discrete cosine and Hartley transforms," IEEE Transactions on Circuits and Systems I: Regular Papers, vol. 60, no. 4, pp. 989-1002, 2012.

[30] M. Jridi, A. Alfalou, and P. K. Meher, "A generalized algorithm and reconfigurable architecture for efficient and scalable orthogonal approximation of DCT," IEEE Transactions on Circuits and Systems I: Regular Papers, vol. 62, no. 2, p. 449-457, 2015.

[31] K. Lengwehasatit and A. Ortega, "Scalable variable complexity approximate forward DCT," IEEE Transactions on Circuits and Systems for Video Technology, vol. 14, no. 11, p. 1236-1248, 2004.

[32] R. T. Haweel, W. S. El-Kilani, and H. H. Ramadan, "Fast approximate DCT with GPU implementation for image compression," Journal of Visual Communication and Image Representation, vol. 40, p. 357-365, 2016.

[33] T. L. da Silveira, R. S. Oliveira, F. M. Bayer, R. J. Cintra, and A. Madanayake, "Multiplierless 16-point DCT approximation for low-complexity image and video coding," Signal, Image and Video Processing, vol. 11, no. 2, p. 227-233, 2017.

[34] J. Huang, T. N. Kumar, H. A. Almurib, and F. Lombardi, "A deterministic low-complexity approximate (multiplier-less) technique for DCT computation," IEEE Transactions on Circuits and Systems I: Regular Papers, vol. 66, no. 8, pp. 3001-3014, 2019. 
[35] H. A. Almurib, T. N. Kumar, and F. Lombardi, "Approximate DCT image compression using inexact computing," IEEE Transactions on computers, vol. 67, no. 2, pp. 149-159, 2017.

[36] C. Tablada, T. L. T. da Silveira, R. J. Cintra, and F. M. Bayer, "DCT approximations based on Chen's factorization," Signal Processing: Image Communication, vol. 58, p. 14-23, 2017.

[37] R. S. Oliveira, R. J. Cintra, F. M. Bayer, T. L. da Silveira, A. Madanayake, and A. Leite, "Low-complexity 8-point DCT approximation based on angle similarity for image and video coding," Multidimensional Systems and Signal Processing, vol. 30, no. 3, p. 1363-1394, 2019.

[38] D. R. Canterle, T. L. da Silveira, F. M. Bayer, and R. J. Cintra, "A multiparametric class of low-complexity transforms for image and video coding," Signal Processing, vol. 176, p. 107685, 2020.

[39] T. I. Haweel, "A new square wave transform based on the DCT," Signal Processing, vol. 81, no. 11, p. 2309-2319, 2001.

[40] N. S. Jayant and P. Noll, "Digital coding of waveforms: principles and applications to speech and video," Englewood Cliffs, NJ, p. 115-251, 1984.

[41] J. Takala and J. Nikara, "Unified pipeline architecture for discrete sine and cosine transforms of type IV," in Proceedings of the 3rd Internacional Conference on Information Communication and Signal Processing, 2001.

[42] K. R. Rao and P. C. Yip, The transform and data compression handbook, vol. 1. CRC press, 2000

[43] J. Katto, K. Komatsu, and Y. Yasuda, "Short-tap and linear-phase PR filter banks for subband coding of images," in Visual Communications and Image Processing'92, vol. 1818, p. 735-747, International Society for Optics and Photonics, 1992

[44] G. A. Seber, A matrix handbook for statisticians, vol. 15. John Wiley \& Sons, 2008.

[45] D. A. Harville, "Trace of a (square) matrix," in Matrix Algebra From a Statistician's Perspective, p. 49-53, Springer, 1997.

[46] R. E. Blahut, Fast algorithms for signal processing. Cambridge University Press, 2010.

[47] A. V. Oppenheim and R. W. Schafer, Digital Signal Processing:(by) Alan V. Oppenheim (and) Ronald W. Schafer. Prentice-Hall, 1975.

[48] A. Levitin, Introduction To Design And Analysis Of Algorithms, 2/E. Pearson Education India, 2008.

[49] C. Loeffler, A. Ligtenberg, and G. S. Moschytz, "Practical fast 1-D DCT algorithms with 11 multiplications," in Acoustics, Speech, and Signal Processing, 1989. ICASSP-89., 1989 International Conference on, p. 988-991, IEEE, 1989.

[50] W.-H. Chen, C. Smith, and S. Fralick, "A fast computational algorithm for the discrete cosine transform," IEEE Transactions on Communications, vol. 25, no. 9, p. 1004-1009, 1977.

[51] F. M. Bayer, R. J. Cintra, A. Edirisuriya, and A. Madanayake, "A digital hardware fast algorithm and FPGA-based prototype for a novel 16-point approximate DCT for image compression applications," Measurement Science and Technology, vol. 23, no. 11, p. 114010, 2012.

[52] F. M. Bayer and R. J. Cintra, "DCT-like transform for image compression requires 14 additions only," Electronics Letters, vol. 48, no. 15, p. 919-921, 2012.

[53] S. Bouguezel, M. O. Ahmad, and M. Swamy, "A fast $8 \times 8$ transform for image compression," in Microelectronics (ICM), 2009 International Conference on, p. 74-77, IEEE, 2009.

[54] S. Bouguezel, M. O. Ahmad, and M. N. S. Swamy, "A low-complexity parametric transform for image compression," in IEEE International Symposium of Circuits and Systems (ISCAS), p. 2145-2148, 2011.

[55] R. J. Cintra, "An integer approximation method for discrete sinusoidal transforms," Circuits, Systems, and Signal Processing, vol. 30, no. 6, p. 1481, 2011.

[56] T. L. da Silveira, F. M. Bayer, R. J. Cintra, S. Kulasekera, A. Madanayake, and A. J. Kozakevicius, "An orthogonal 16-point approximate dct for image and video compression," Multidimensional Systems and Signal Processing, vol. 27, no. 1, pp. 87-104, 2016.

[57] A. Gupta and K. R. Rao, "A fast recursive algorithm for the discrete sine transform," IEEE Transactions on Acoustics, Speech, and Signal Processing, vol. 38 , no. 3, pp. 553-557, 1990.

[58] Joint Collaborative Team on Video Coding (JCT-VC), "HEVC reference software documentation," 2013. Fraunhofer Heinrich Hertz Institute.

[59] D. Salomon, Data compression: the complete reference. Springer Science \& Business Media, 2004.

[60] Q. Huynh-Thu and M. Ghanbari, "Scope of validity of PSNR in image/video quality assessment," Electronics Letters, vol. 44, no. 13, p. 800-801, 2008.

[61] Z. Wang, A. C. Bovik, H. R. Sheikh, and E. P. Simoncelli, "Image quality assessment: from error visibility to structural similarity," IEEE Transactions on Image Processing, vol. 13, no. 4, p. 600-612, 2004.

[62] U. SIPI, "The USC-SIPI image database," 1977.

[63] J.-R. Ohm, G. J. Sullivan, H. Schwarz, T. K. Tan, and T. Wiegand, "Comparison of the coding efficiency of video coding standards - including High Efficiency Video Coding (HEVC)," IEEE Transactions on Circuits and Systems for Video Technology, vol. 22, pp. 1669-1684, Dec. 2012.

[64] D. J. Le Gall, "The MPEG video compression algorithm," Signal Processing: Image Communication, vol. 4, no. 2, pp. 129-140, 1992.

[65] International Telecommunication Union, "ITU-T recommendation H.261 version 1: Video codec for audiovisual services at $p \times 64 \mathrm{kbits}$," tech. rep., ITU-T, 1990.

[66] International Telecommunication Union, "ITU-T recommendation H.263 version 1: Video coding for low bit rate communication," tech. rep., ITU-T, 1995.

[67] A. Luthra, G. J. Sullivan, and T. Wiegand, "Introduction to the special issue on the H.264/AVC video coding standard," IEEE Transactions on Circuits and Systems for Video Technology, vol. 13, pp. 557-559, July 2003.

[68] S. Harize, H. Semira, M. Benouaret, and N. Doghmane, "Hardware implementation and performance evaluation of the hevc intra-predicted $4 \backslash \times 4$ blocks transforms," Circuits, Systems, and Signal Processing, vol. 36, no. 5, pp. 2050-2074, 2017.

[69] F. Bossen, "Common test conditions and software reference configurations," Feb 2013. Document JCT-VC L1100.

[70] X. video test media, "Xiph.org foundation," 2014.

[71] G. Bjøntegaard, "Calculation of average PSNR differences between RD-curves," in 13th VCEG Meeting, (Austin, TX, USA), Apr 2001. Document VCEG-M33.

[72] P. Hanhart and T. Ebrahimi, "Calculation of average coding efficiency based on subjective quality scores," Journal of Visual Communication and Image Representation, vol. 25, no. 3, pp. 555 - 564, 2014. QoE in 2D/3D Video Systems.

[73] Y. Zeng, H. Sun, J. Katto, and Y. Fan, "Approximated reconfigurable transform architecture for vvc," in 2021 IEEE International Symposium on Circuits and Systems (ISCAS), pp. 1-5, IEEE, 2021.

[74] W. Imen, B. Fatma, M. Amna, and N. Masmoudi, "DCT-II transform hardware-based acceleration for vvc standard," in 2021 IEEE International Conference on Design \& Test of Integrated Micro \& Nano-Systems (DTS), pp. 1-5, IEEE, 2021.

[75] K. Ding, D. Jiang, F. Zeng, J. Lin, and J. Yin, "A fast transform algorithm based on vvc," in 2020 4th International Conference on Computer Science and Artificial Intelligence, pp. 80-85, 2020. 